\title{
Fibroblasts Promote Inflammation and Pain via IL-1 $\alpha$ Induction of the Monocyte Chemoattractant Chemokine (C-C Motif) Ligand 2
}

Hannah L. Paish, ${ }^{*}$ Nicholas S. Kalson, ${ }^{* \dagger}$ Graham R. Smith, ${ }^{*}$ Alicia del Carpio Pons, ${ }^{*}$ Thomas E. Baldock, ${ }^{\dagger}$ Nicholas Smith, ${ }^{*}$ Katarzyna Swist-Szulik, ${ }^{\S}$ David J. Weir, ${ }^{\dagger}$ Michelle Bardgett, ${ }^{\dagger}$ David J. Deehan, ${ }^{* \dagger}$ Derek A. Mann, ${ }^{*}$ and Lee A. Borthwick

From the Fibrosis Research Group, * Institute of Cellular Medicine, the Bioinformatics Support Unit, ${ }^{\ddagger}$ and the Wellcome Centre for Mitochondrial Research, ${ }^{\S}$ Institute of Neuroscience, Newcastle University, Newcastle upon Tyne; and the Musculoskeletal Unit, ${ }^{\dagger}$ Freeman Hospital, Newcastle Hospitals, NHS Trust, Newcastle upon Tyne, United Kingdom

\author{
Accepted for publication \\ November 9, 2017. \\ Address correspondence to \\ Lee A. Borthwick, Ph.D., \\ Fibrosis Research Group, Insti- \\ tute of Cellular Medicine, \\ Newcastle University, Fram- \\ lington Place, Tyne and Wear \\ NE2 4HH, United King- \\ dom. E-mail: lee.borthwick@ \\ newcastle.ac.uk.
}

\begin{abstract}
Fibroblasts persist within fibrotic scar tissue and exhibit considerable phenotypic and functional plasticity. Herein, we hypothesized that scar-associated fibroblasts may be a source of stressinduced inflammatory exacerbations and pain. To test this idea, we used a human model of surgery-induced fibrosis, total knee arthroplasty (TKA). Using a combination of tissue protein expression profiling and bioinformatics, we discovered that many months after TKA, the fibrotic joint exists in a state of unresolved chronic inflammation. Moreover, the infrapatellar fat pad, a soft tissue that becomes highly fibrotic in the post-TKA joint, expresses multiple inflammatory mediators, including the monocyte chemoattractant, chemokine ( $\mathrm{C}-\mathrm{C}$ motif) ligand $(\mathrm{CCL}) 2$, and the innate immune trigger, IL-1 $\alpha$. Fibroblasts isolated from the post-TKA fibrotic infrapatellar fat pad express the IL-1 receptor and on exposure to IL-1 $\alpha$ polarize to a highly inflammatory state that enables them to stimulate the recruitment of monocytes. Blockade of fibroblast CCL2 or its transcriptional regulator NF- $\kappa B$ prevented IL-1 $\alpha$-induced monocyte recruitment. Clinical investigations discovered that levels of patient-reported pain in the post-TKA joint correlated with concentrations of CCL2 in the joint tissue, such that the chemokine is effectively a pain biomarker in the TKA patient. We propose that an IL-1 $\alpha-N F-\kappa B-C C L 2$ signaling pathway, operating within scar-associated fibroblasts, may be therapeutically manipulated for alleviating inflammation and pain in fibrotic joints and other tissues. (Am J Pathol 2018, 188: 696-714; https://doi.org/ 10.1016/j.ajpath.2017.11.007)
\end{abstract}

Fibrosis is the excessive deposition of scar tissue arising within a tissue or joint after injury or trauma. ${ }^{1}$ Adhesions are fibrous bands that are generated after surgery; they form physical connections between tissues that are not normally directly connected, resulting in derangement and loss of function of the affected tissue or joint. ${ }^{2}$ Fibrous adhesions can also be associated with intermittent periods of acute inflammation, debilitating pain, and progressive degeneration of the affected tissue or joint. ${ }^{3}$ Therapeutic modulation of these acute exacerbations is limited by our current poor understanding of their underlying molecular triggers and mediators.
Supported by the Medical Research Council grant MR/K001949/1, Wellcome Trust grant 204787/Z/16/Z, Newcastle Surgical Training Center grant, The Royal College of Surgeons of Edinburgh grant, and the National Institute for Health Research Newcastle Biomedical Research Centre grant, based at Newcastle-upon-Tyne Hospitals NHS Foundation Trust and Newcastle University.

D.A.M. and L.A.B. contributed equally to this work.

Disclosures: D.A.M. has acted as a consultant for Abbvie and GlaxoSmithKline, is a scientific advisor for Bird Rock Bio, and has received grant support from Abbvie and GlaxoSmithKline.

The views expressed herein are those of the author and not necessarily those of the NHS, the National Institute for Health Research, or the Department of Health 
Table 1 Patient Demographics

\begin{tabular}{|c|c|c|c|c|c|}
\hline Variable & $\begin{array}{l}\text { Sex, } \\
\text { M:F ratio }\end{array}$ & $\begin{array}{l}\text { Age in years, } \\
\text { median (range) }\end{array}$ & $\begin{array}{l}\text { BMI, } \\
\text { median (range) }\end{array}$ & $\begin{array}{l}\text { Time in months from } \\
\text { primary to revision TKA, } \\
\text { median (range) }\end{array}$ & $\begin{array}{l}\text { Primary indication } \\
\text { for surgery }\end{array}$ \\
\hline Primary TKA $(n=29)$ & $17: 12$ & $66(44-81)$ & $32(22-47)$ & NA & $\begin{array}{l}\text { Osteoarthritis }(n=28), \\
\text { rheumatoid disease }(n=1)\end{array}$ \\
\hline Revision TKA $(n=33)$ & $16: 17$ & $71(40-88)$ & $33(23-46)$ & $106(8-360)$ & $\begin{array}{l}\text { Osteolysis and loose } \\
\text { components }(n=11) \text {, } \\
\text { clinical diagnosis of fibrosis with } \\
\text { loss of movement ( } n=11) \text {, } \\
\text { primary laxity pattern with functional } \\
\text { instability }(n=8) \text {, } \\
\text { progression of osteoarthritis } \\
\text { (previous patellofemoral resurfacing) } \\
(n=3)\end{array}$ \\
\hline
\end{tabular}

F, female; M, male; BMI, body mass index; NA, not applicable; TKA, total knee arthroplasty.

The cell type responsible for the deposition of fibrotic extracellular matrix is the fibroblast. ${ }^{4,5}$ After tissue damage, resident pericytes, fibroblasts, or epithelial cells transdifferentiate to the fibroblast phenotype characterized by their expression of $\alpha$-smooth muscle actin and abundant secretion of collagens. ${ }^{6}$ Resolution of wound healing is associated with the clearance of fibroblasts, whereas the maintenance and progression of fibrosis is associated with fibroblast proliferation and survival. ${ }^{7}$ Fibroblasts exhibit considerable phenotypic plasticity and may even reverse transdifferention or alternatively adopt an inactivated state characterized by reduced secretion of extracellular matrix proteins and an extended lifespan of several weeks. ${ }^{89}$ An often underappreciated feature of fibroblasts is their ability to adopt an immune effector phenotype in response to stimulation with IL- $1 \alpha / \beta$ or tumor necrosis factor- $\alpha .^{10-13}$ We recently described how epithelial stress, via release of IL-1 $\alpha$, triggers the polarization of lung fibroblasts toward a proinflammatory phenotype characterized by their highly abundant secretion of IL-6, IL-8, chemokine (C-C motif) ligand (CCL) 2 (monocyte chemoattractant protein-1), and granulocyte-macrophage colony-stimulating factor. ${ }^{14,15}$ Similar inflammatory cytokine release has also been described in hepatic stellate cells, ${ }^{16}$ cardiac fibroblasts, ${ }^{17}$ dermal fibroblasts, ${ }^{18}$ and fibroblasts isolated from the knee, ${ }^{19}$ suggesting the ability to adopt an immunomodulatory state may be a ubiquitous property of fibroblasts. Herein, we hypothesized that fibroblasts persisting within fibrotic microenvironments may be a source of stressinduced inflammatory exacerbations and pain.

To test this hypothesis, we used a human model of surgeryinduced fibrosis, the post-surgical knee. Total knee arthroplasty (TKA) is a proven cost-effective treatment for painful end-stage knee osteoarthritis, in which the articulating joint surfaces are removed and replaced with new prostheticbearing surfaces. ${ }^{20,21}$ After surgery, the TKA knee is characterized by a fibrotic process, the persistence of fibroblasts lasting months after surgery, and the deposition of vast quantities of fibrotic extracellular matrix. ${ }^{22,23}$ Furthermore, approximately $20 \%$ of TKA patients report swelling and pain of their joint that can be more severe than that experienced before surgery. ${ }^{24,25}$ By acquiring synovial fluid and tissue from patients undergoing primary TKA (nonfibrotic) and patients undergoing revision TKA (fibrotic), we were able to define the inflammatory profile of the fibrotic joint and a role for IL-1 receptor (IL-1R1), expressing inflammatory fibroblasts. Using a combination of bioinformatics and in vitro assays, we provide evidence that inflammatory fibroblasts regulate monocyte recruitment to fibrotic tissues via the release of CCL2 and demonstrate that CCL2 levels in the post-TKA joint correlate with reported pain.

\section{Materials and Methods}

\section{Ethics}

This study was performed in accordance with approval from the Newcastle and North Tyneside Local Regional Ethics Committee, and informed written consent was obtained from all patients (12/NE/0395). Samples used in this research were obtained from the Newcastle Biomedicine Biobank (Newcastle upon Tyne, UK; http://www.ncl.ac.uk/ nbb/collections).

\section{Tissue Collection and Patient Stratification}

All patients undergoing revision surgery for failed primary TKA were included in the study during a 2-year period, with infection being the only exclusion criterion. Synovial fluid, infrapatellar fat pad, and synovial membrane (suprapatellar pouch) were collected from patients undergoing either primary $(n=29)$ or revision $(n=33)$ TKA at the Freeman Hospital (Newcastle upon Tyne, UK) (Table 1).

Revision TKA patients were stratified as those with osteolysis and loose components $(n=11)$, a clinical diagnosis of fibrosis with loss of movement $(n=11)$, primary laxity pattern with functional instability $(n=8$, of which $n=6$ had lost posterior cruciate ligament-required 
revision to a higher constraint prosthesis and $n=2$ had midpoint laxity and the collateral ligaments were lax in full extension and underwent revision to a higher constraint implant), and progression of osteoarthritis (previous patellofemoral resurfacing) $(n=3)$. In the revision cohort, the primary knee replacements were as follows: 16 PFC (Sigma, St. Louis, MO), six Kinemax (Howmedica Osteonics Corporation, Mahwah, NJ), two Triathlon (Stryker, Kalamazoo, MI), one Genesis II (Smith and Nephew, London, UK), one Oxford unicompartmental (Zimmer, Warsaw, IN), one Vanguard (Biomet, Warsaw, IN), one AGC (Biomet), one IB2 (Zimmer), one Noiles (DePuy, Raynham, MA), and three primary patella-femoral resurfacing. Six revision patients with a total knee replacement in situ also had a patella-femoral resurfacing.

For the purposes of our study, we used a definition of primary joint fibrosis as previously described. ${ }^{22,26}$ In all primary and revision TKA patients, local anesthetic was administered intraoperatively under direct vision (to a maximum dose of $100 \mathrm{~mL}$ of $0.2 \%$ ropivacaine). All patients were given routine postoperative analgesia, including regular paracetamol and a patient-controlled analgesia device. In addition, patients with restricted range of motion (fibrotic revisions) underwent continuous passive movement for 48 to 72 hours postoperatively.

Routine practice in our unit includes excision of the infrapatellar fat pad. All primary patients in the study had their infrapatellar fat pad removed. In the revision cohort, 24 had primary surgery performed in our unit and, therefore, had their infrapatellar fat pad removed in the primary procedure. The remaining nine revision patients had surgery elsewhere, and we do not know whether the infrapatellar fat pad was removed in their primary procedure. The infrapatellar fat pad tissue resected in revision surgery was from an anatomically matched site to the normal fat pad tissue found in the primary knee and resected in primary replacement surgery.

Synovial fluid was collected by aspirating fluid from the knee during surgery. The sample was sterile filtered $(0.4 \mu \mathrm{m}$ thick $)$ and stored at $-80^{\circ} \mathrm{C}$. Representative tissue biopsy specimens from infrapatellar fat pad and synovial membrane were fixed in formalin and paraffin embedded. Serial tissue sections $(5 \mu \mathrm{m}$ thick) were cut and processed for staining, as described later. Tissue was homogenized in radioimmunoprecipitation assay buffer supplemented with protease and phosphatase inhibitors using a bead homogenizer (TissueLyserII; Qiagen, Hilden, Germany). Homogenized samples were normalized to $1 \mathrm{mg} / \mathrm{mL}$ total protein (protein concentration measured using a bicinchoninic acid protein assay; Pierce, Waltham, MA), as per manufacturer's instructions, and stored at $-80^{\circ} \mathrm{C}$

\section{Exclusion of Infection}

Preoperative workup included C-reactive protein (all patients) and joint aspiration (7 of 33 revision patients). Joint aspiration was performed only when the treating clinician believed that infection needed to be excluded. C-reactive protein was $<30$ in all revision patients, and all preoperative joint aspiration samples were negative after extended culture. Infection was definitively ruled out in all revision patients by microbiological analysis of synovial fluid and of multiple tissue samples taken at revision surgery. All revision patients had negative extended culture of both intraoperative synovial fluid and tissue samples. A minimum of three and a maximum of six tissue samples were taken intraoperatively, according to prosthetic joint infection guidelines. ${ }^{27}$ As is standard practice, all orthopedic theater samples underwent extended enrichment culture for 10 days. However, a role for organisms, such as Propionibacterium acnes, that are challenging to culture, and may be an underrecognized cause of knee prosthetic joint infection, cannot be ruled out. ${ }^{28,29}$

\section{Histological Analysis of Tissue Samples}

Formal histology reports were available for 9 of 33 revision patients. All reports state that dense, hypocellular, heavily collagenized fibrous tissue foreign body giant cells containing small transparent, strongly birefringent foreign particles were identified. The appearances are those of extensive fibrosis together with a focal giant cell reaction to foreign material, a reaction to polyethylene debris from the prosthetic joint implant. There was no evidence of infection reported in any cases.

\section{Immunohistochemistry}

Tissue sections (5 $\mu \mathrm{m}$ thick) were deparaffinized in xylene, rehydrated in graded alcohol, and incubated in $0.6 \%$ hydrogen peroxide/methanol for 15 minutes. Antigen retrieval was performed using $20 \mu \mathrm{g} / \mathrm{mL}$ Proteinase $\mathrm{K}$ (Sigma) in phosphate-buffered saline (PBS) at $37^{\circ} \mathrm{C}$ for 30 minutes. Blocking was performed using Avidin/Biotin Block (Vector Laboratories, Burlingame, CA), followed by porcine serum. Primary antibody incubation was performed overnight at $4^{\circ} \mathrm{C}$ using rabbit polyclonal primary antibody against IL1R1 (Ab106278; Abcam, Cambridge, UK). Slides were washed with PBS and incubated with anti-rabbit biotinylated antibody $(0.51 \mathrm{mg} / \mathrm{L}$; Dako, Santa Clara, CA) in $1 \%$ porcine serum for 1 hour at room temperature. ABC tertiary (Vector Laboratories) was then applied to slides for 45 minutes at room temperature before application of 3,3'-diaminobenzidine mix (Vector Laboratories) for 5 minutes, followed by counterstaining with Mayer's hematoxoylin (Sigma). Slides were dehydrated in graded ethanol and Clearene (Leica Biosystems, Wetzlar, Germany) before mounting using Pertex (CellPath Ltd., Newtown Powys, UK). Images were acquired using a Nikon Eclipse Upright Microscope (Nikon Instruments Europe BV, Amsterdam, the Netherlands). For CD68 image quantification, the mean number of positive cells in $\times 10$ randomly selected highpowered fields $(\times 20$ magnification) was calculated using Nikon NIS elements image analysis software version 4.0 (Nikon Instruments Europe BV). 


\section{Immunocytochemistry}

Tissue sections ( $5 \mu \mathrm{m}$ thick) were deparaffinized in xylene and rehydrated in graded alcohol. Antigen retrieval was performed with proteinase $\mathrm{K}(20 \mu \mathrm{g} / \mathrm{mL}$ in PBS) for 30 minutes at $37^{\circ} \mathrm{C}$. Samples were blocked with $5 \%$ bovine serum albumin/PBS and incubated overnight at $4{ }^{\circ} \mathrm{C}$ with primary antibodies diluted in 5\% bovine serum albumin/ PBS [IL-1R1, ab106278 (Abcam, Cambridge UK); IL-6, AF-206 at $10 \mu \mathrm{g} / \mathrm{mL}$ (R\&D Systems, Minneapolis, MN); vimentin, M7020 at $62 \mu \mathrm{g} / \mathrm{mL}$ (Dako)]. Slides were washed with PBS-Tween (0.05\%) and incubated with anti-rabbit tetrarhodamine isothiocyanate (T6778; SigmaAldrich) and anti-mouse fluorescein isothiocyanate (F2012; Sigma-Aldrich) diluted in 5\% bovine serum albumin/PBS for 1 hour at room temperature. Slides were washed with PBS-Tween and mounted using mounting medium with DAPI (H1200; Vector Laboratories). Images were acquired on a Nikon A1R point scanning confocal microscope.

\section{Multiarray Protein Assays}

Synovial fluid (primary, $n=21$; revision, $n=24$ ) and tissue homogenates from infrapatellar fat pad (primary, $n=28$ patients; revision, $n=32$ patients) and synovial membrane (primary, $n=29$ patients; revision, $n=32$ patients) were assessed for expression of 39 protein markers using a human V-Plex electrochemiluminescence detection kit (K15209D; MesoScaleDiscovery, Rockville, $\mathrm{MD}$ ), as per manufacturer's instructions. Analysis of results was performed using the MSD Discovery Workbench software version 4.0 (MesoScaleDiscovery, Rockville, MD).

\section{Network Reconstruction}

To identify potentially important intracellular signaling pathways/processes likely occurring in the revision TKA knee, we constructed a network using protein-protein interactions from InnateDB (http://www.innatedb.com). The differentially expressed markers and $\log$ (fold changes) were uploaded to the web server NetworkAnalyst (http:// www.networkanalyst.ca), ${ }^{30}$ and the network was built with direct interactions only from the innate DB interactome. ${ }^{31}$ The resulting network contains 730 nodes and 921 edges and was visualized with Cytoscape. ${ }^{32}$ Statistics, such as degree (number of connections of a given node) and betweenness centrality (the number of paths between other nodes passing through a given node), were calculated. Modules (highly connected subnetworks) were found by a method based on the spreading rate of a random walk on the network. Significant gene ontology terms were identified using overrepresentation of the proteins in the network, or modules of the network, among gene sets annotated with these terms.

\section{Fibroblast Isolation}

Infrapatellar fat pad isolated from patients undergoing primary and revision TKA was homogenized and digested in $10 \mathrm{~mL}$ of supplement-free medium [Dulbecco's modified Eagle's medium (DMEM); D5671; Sigma] containing 100 units $/ \mathrm{mL}$ of collagenase (C6885; Sigma) for 60 minutes. Digested tissue was passed through a $100-\mu \mathrm{m}$ filter, and the collagenase was neutralized with complete medium (DMEM plus $10 \%$ fetal calf serum, $100 \mu \mathrm{g} / \mathrm{mL}$ streptomycin, $100 \mathrm{U} / \mathrm{mL}$ penicillin, and $1 \%$ L-glutamate). Cells were pelleted and resuspended in complete medium before transferring into T-75 flasks. Cells were cultured at $37^{\circ} \mathrm{C}$ and $5 \% \mathrm{CO}_{2}$ until $>90 \%$ confluent. Cells were cryopreserved at passage number $(\mathrm{P}) 0$ or passaged and cryopreserved at P1. Mesenchymal phenotype was confirmed, as previously described. ${ }^{19}$ Fibroblasts were reanimated and used between P2 and P5 for all experiments in this study.

\section{Cell Treatments}

Fibroblasts isolated from the infrapatellar fat pad of patients undergoing primary ( $n=6$ patients) or revision ( $n=6$ patients) TKA were serum starved for 24 hours before treatment with $0 \%$ fetal bovine serum (FBS) DMEM (control), 0\% FBS DMEM + IL- $1 \alpha(500 \mathrm{pg} / \mathrm{mL})$, or 0\% FBS DMEM + transforming growth factor (TGF)- $\beta 1$ (3 ng/mL) for 24 hours. In some experiments, fibroblasts were pretreated with inhibitors [SP600125， SD1008， FR180204， SB203580, (5Z)-7oxozeaenol, IL-1 receptor associated kinase 1/4 inhibitor 1 (all from Tocris, Minneapolis, MN) or I $\mathrm{B}$ kinase (IKK)-2 inhibitor VIII (MerckMillipore, Billerica, MA)] for 1 hour before treatment with inhibitors $+\mathrm{IL}-1 \alpha(500 \mathrm{pg} / \mathrm{mL})$. After 24 hours, the medium was collected, spun at $800 \times g$ to clear, and stored at $-80^{\circ} \mathrm{C}$ for analysis or use in transwell migration assays.

\section{Enzyme-Linked Immunosorbent Assay}

IL-6, IL-8, and CCL2 protein levels in media samples from fibroblasts were quantified by ELISA (R\&D Systems), as per manufacturer's instructions.

\section{Lactate Dehydrogenase Assay}

Lactate dehydrogenase levels in media collected from fibroblasts were quantified using a Pierce LDH Cytotoxicity Assay Kit (Thermo Scientific, Waltham, MA), as per manufacturer's protocol.

\section{THP-1 Cell}

THP-1 cells are an acute monocytic leukemia human cell line and were purchased from ATCC (TIB-202; ATCC, Manassas, VA). Cells were cultured in RPMI-1640 media (R0883; Sigma) supplemented with 10\% FBS, 1\% L-glutamine, $100 \mathrm{U} / \mathrm{mL}$ penicillin, and $100 \mu \mathrm{g} / \mathrm{mL}$ streptomycin. Cells were maintained at approximately $0.5 \times 10^{6}$ cells $/ \mathrm{mL}$, 
A

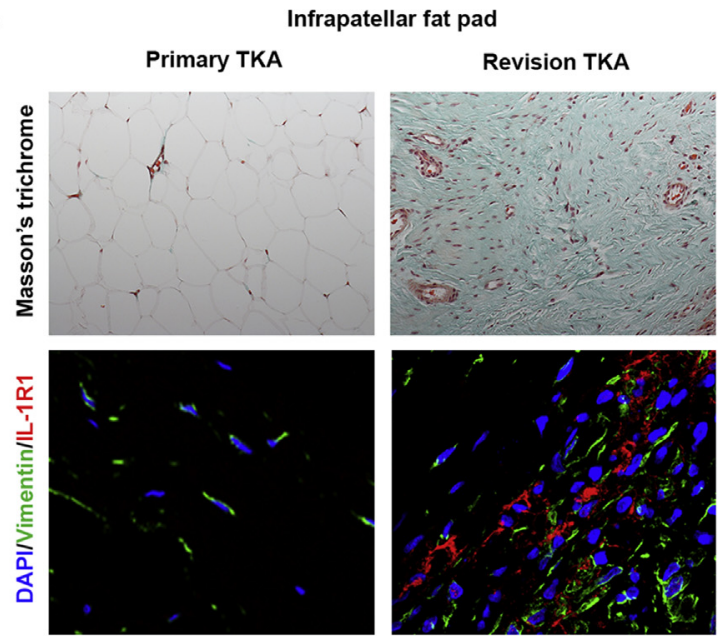

B
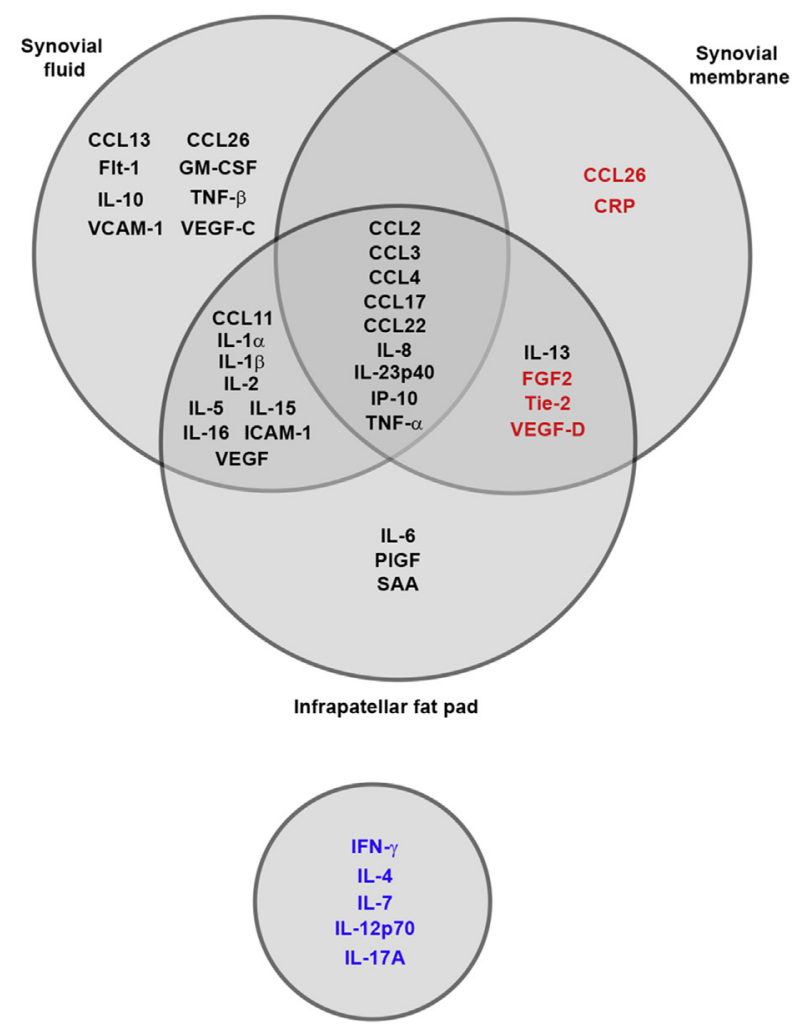

Unchanged or undetectable

C Revision TKA - infrapatellar fat pad
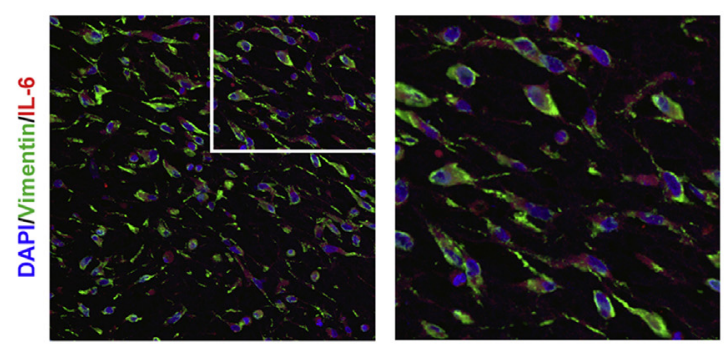

and medium was changed twice weekly. THP-1 cells were used to provide a uniform monocyte population and ensure the only experimental variable in transwell migration assays was the composition of the conditioned media.

\section{Transwell Migration Assay}

Media from fibroblasts treated with 0\% FBS DMEM (control), $0 \%$ FBS DMEM + IL-1 $\alpha(500 \mathrm{pg} / \mathrm{mL}), 0 \%$ FBS DMEM + TGF- $\beta 1(3 \mathrm{ng} / \mathrm{mL})$, or fibroblasts pretreated with inhibitors before treatment with 0\% FBS DMEM + IL-1 $\alpha$ were placed directly into the bottom of a 24-well plate. Recombinant CCL2 $(100 \mathrm{pg} / \mathrm{mL})$ in 0\% FBS DMEM was used as a positive control, and 0\% FBS DMEM was used as a negative control. THP- 1 cells $(50,000$ in $100 \mu \mathrm{L} 0 \%$ FBS DMEM) were added to a $6.5-\mathrm{mm}, 5-\mu \mathrm{m}$ pore transwell insert (Costar Corning, Corning, NY), and the insert was added to the 24-well plate. After 18 hours, migrated cells were imaged using a Nikon D5000 camera and quantified using ImageJ software version 1.41 (NIH, Bethesda, MD; http://imagej.nih.gov/ij). In some experiments, medium from fibroblasts treated with $0 \%$ FBS DMEM + IL- $1 \alpha(500 \mathrm{pg} / \mathrm{mL})$ was pretreated with neutralizing antibodies against CCL2 $(4 \mu \mathrm{g} / \mathrm{mL}$; AF-479; R\&D Systems) and IL-6 (200 $\mu \mathrm{g} / \mathrm{mL}$; Ab6672; Abcam) for 1 hour before adding to the 24-well plate.

\section{Clustering and Stratification}

In the clustering analysis, protein expression values were transformed according to $\log 10$ (intensity $+2 * \delta$ ), where $\delta$ is

Figure 1 Synovial fluid and tissues isolated from patients undergoing revision total knee arthroplasty (TKA) are characterized by dramatic tissue remodeling and chronic inflammation. A: Representative images of Masson trichrome and IL-1R1 (green [fluorescein isothiocyanate (FITC)])/vimentin (red [tetrarhodamine isothiocyanate (TRITC)]) stained infrapatellar fat pad from patients undergoing primary TKA and patients undergoing revision TKA. There is a significant increase in collagen and IL-1R1 expression in revision TKA tissue compared with primary TKA tissue, and IL-1R1 is predominantly expressed on elongated bipolar mesenchymal cells in revision TKA tissue. Images acquired on a Nikon inverted microscope and a Nikon A1R point scanning confocal microscope, respectively. B: Venn diagram demonstrating change in protein expression in synovial fluid, infrapatellar fat pad, and synovial membrane isolated from patients undergoing revision TKA compared with patients undergoing primary TKA. Protein expression was quantified using human V-Plex electrochemiluminescence detection kits from MesoScaleDiscovery. Markers in black text are significantly elevated in revision TKA, markers in blue text are not significantly different or undetectable, and markers in red text are significantly decreased in revision TKA. Significance was taken as $P<0.05$. C: Representative images of vimentin [green (FITC)]- and IL-6 [red (TRITC)]-stained infrapatellar fat pad from patients undergoing revision TKA. There are a significant number of vimentin and IL- 6 dual-positive fibroblasts in post-TKA infrapatellar fat pad. Images acquired on a Nikon A1R point scanning confocal microscope. Original magnification, $\times 20$ (A and C); $\times 40$ (inset). CRP, Creactive protein; FGF, fibroblast growth factor; Flt, Fms-like tyrosine kinase 1; GM-CSF, granulocyte-macrophage colony-stimulating factor; ICAM, intercellular adhesion molecule; IFN, interferon; IP-10, interferon gammainduced protein 10; PIGF, placental growth factor; SAA, serum amyloid $A_{\text {; }}$ Tie, tyrosine kinase; TNF, tumor necrosis factor; VCAM, vascular cell adhesion molecule; VEGF, vascular endothelial growth factor. 
Table 2 Synovial Fluid: Primary TKA versus Revision TKA

\begin{tabular}{|c|c|c|c|c|c|c|}
\hline \multirow[b]{2}{*}{ Marker } & \multicolumn{2}{|l|}{ Primary TKA } & \multicolumn{2}{|l|}{ Revision TKA } & \multicolumn{2}{|c|}{ Revision versus Primary TKA } \\
\hline & Median, $\mathrm{pg} / \mathrm{mL}$ & Range, $\mathrm{pg} / \mathrm{mL}$ & Median, $\mathrm{pg} / \mathrm{mL}$ & Range, $\mathrm{pg} / \mathrm{mL}$ & Fold change & $P$ value \\
\hline \multicolumn{7}{|c|}{ Significantly increased } \\
\hline CCL2 & 395 & $67-857$ & 3744 & $318-23,297$ & 9.48 & $<0.0001$ \\
\hline CCL3 & 13 & $0-51$ & 102 & $15-1257$ & 7.93 & $<0.0001$ \\
\hline IL-10 & 0.16 & $0-11.2$ & 0.92 & $0-8.3$ & 5.58 & $<0.0001$ \\
\hline GM-CSF & 0.19 & $0-4.5$ & 0.71 & $0-4.0$ & 3.71 & $<0.01$ \\
\hline CCL13 & 15.0 & $9-415$ & 54.8 & $14-1320$ & 3.67 & $<0.0001$ \\
\hline IP-10 & 256 & $130-9980$ & 873 & $196-5562$ & 3.41 & $<0.001$ \\
\hline CCL4 & 60 & $27-195$ & 200 & $36-1358$ & 3.33 & $<0.0001$ \\
\hline TNF- $\alpha$ & 1.16 & $0.5-5.9$ & 3.81 & $0.8-28.1$ & 3.30 & $<0.0001$ \\
\hline CCL17 & 28.0 & $11-337$ & 71.7 & $14-384$ & 2.56 & $<0.001$ \\
\hline IL-23p40 & 42.8 & $6-652$ & 90.2 & $11-403$ & 2.11 & $<0.01$ \\
\hline TNF- $\beta$ & 0.28 & $0-2.1$ & 0.59 & $0-9.5$ & 2.08 & $<0.001$ \\
\hline CCL11 & 27.3 & $15-94$ & 56.0 & $23-206$ & 2.05 & $<0.001$ \\
\hline IL-15 & 15.6 & $3-25$ & 27.9 & $9-104$ & 1.79 & $<0.0001$ \\
\hline ICAM-1 & 66,071 & $25,024-265,365$ & 101,434 & $31,383-458,122$ & 1.54 & $<0.05$ \\
\hline VCAM-1 & 100,705 & $31,963-167,370$ & 128,900 & $70,772-210,136$ & 1.28 & $<0.05$ \\
\hline CCL26 & 0.0 & $0-36$ & 13.3 & $0-107$ & NA & $<0.0001$ \\
\hline IL-1 $\beta$ & 0.00 & $0-1.3$ & 0.10 & $0-47.1$ & NA & $<0.001$ \\
\hline IL-1 $\alpha$ & 0.00 & $0-1.4$ & 0.28 & $0-22.9$ & NA & $<0.01$ \\
\hline $\mathrm{IL}-2$ & 0.00 & $0-0.4$ & 0.17 & $0-3.2$ & NA & $<0.05$ \\
\hline CRP & 875,587 & $11,385-1,421,021$ & 902,869 & $25,449-1,865,959$ & 1.03 & 0.72 \\
\hline IFN- $\gamma$ & 2.54 & $0-105$ & 2.53 & $0-55$ & 1.00 & 0.88 \\
\hline SAA & 205,119 & $43,141-851,868$ & 198,230 & $57,469-1,072,174$ & 0.97 & 0.81 \\
\hline IL-7 & 8.49 & $0.7-16.5$ & 7.30 & $2.5-13.6$ & 0.86 & 0.29 \\
\hline FGF2 & 35.2 & $3-512$ & 21.6 & $3-495$ & 0.61 & 0.25 \\
\hline IL-12p70 & 0.00 & $0-1.1$ & 0.00 & $0-7.5$ & NA & 0.11 \\
\hline IL-13 & 0.00 & $0-3.9$ & 0.00 & $0-16.5$ & NA & 0.09 \\
\hline
\end{tabular}

CRP, C-reactive protein; FGF, fibroblast growth factor; Flt, Fms-like tyrosine kinase 1; GM-CSF, granulocyte-macrophage colony-stimulating factor; ICAM, intercellular adhesion molecule; IFN, interferon; IP-10, interferon gamma-induced protein 10; NA, not applicable; PIGF, placental growth factor; SAA, serum amyloid A; Tie, tyrosine kinase; TKA, total knee arthroplasty; TNF, tumor necrosis factor; VCAM, vascular cell adhesion molecule; VEGF, vascular endothelial growth factor.

the smallest nonzero measured value. The measured markers were then sorted by fold change between primary and revision, and the top $n$ values were used for clustering, where $n$ was chosen so that $(n+1)$ is the first not significantly DE between the two groups or where this would lead to few proteins, $n=10$. The distance measure between marker sets was the euclidean distance between the logtransformed expression, and Ward's method was used for hierarchical clustering. Heat maps were generated with the ComplexHeatmap package in $\mathrm{R}$ version 3.2.3 (The $\mathrm{R}$
Foundation for Statistical Computing, Vienna, Austria). For display, each row is $\mathrm{Z}$ transformed to equalize differences in overall expression. Principal component analysis was performed using the base $\mathrm{R}$ function princomp and all markers.

\section{Stratification with a Reduced Set of Markers}

A reduced set of markers was selected with the $\mathrm{R}$ package glmnet (http://www.jstatsoft.org/v33/i01). ${ }^{33}$ The binomial family was used for the generalized linear model, with 
Table 3 Infrapatellar Fat Pad: Primary TKA versus Revision TKA

\begin{tabular}{|c|c|c|c|c|c|c|}
\hline \multirow[b]{2}{*}{ Marker } & \multicolumn{2}{|l|}{ Primary TKA } & \multicolumn{2}{|l|}{ Revision TKA } & \multicolumn{2}{|c|}{ Revision versus Primary TKA } \\
\hline & Median, $\mathrm{pg} / \mathrm{mL}$ & Range, $\mathrm{pg} / \mathrm{mL}$ & Median, pg/mL & Range, $\mathrm{pg} / \mathrm{mL}$ & Fold change & $P$ value \\
\hline \multicolumn{7}{|c|}{ Significantly increased } \\
\hline CCL4 & 2.8 & $0-12$ & 26.0 & $0-522$ & 9.25 & $<0.0001$ \\
\hline CCL2 & 18 & $7-54$ & 142 & $9-1491$ & 8.03 & $<0.0001$ \\
\hline IL-13 & 0.16 & $0-2.2$ & 1.15 & $0-4.0$ & 7.18 & $<0.01$ \\
\hline CCL22 & 41 & $12-125$ & 154 & $21-3827$ & 3.76 & $<0.0001$ \\
\hline $\mathrm{IL}-1 \beta$ & 0.07 & $0-0.3$ & 0.23 & $0-7.6$ & 3.19 & $<0.001$ \\
\hline CCL17 & 1.20 & $0-11$ & 3.51 & $1-27$ & 2.91 & $<0.0001$ \\
\hline VEGF & 22.5 & $0-83$ & 61.5 & $8-1616$ & 2.74 & $<0.0001$ \\
\hline IL-15 & 1.47 & $0-8$ & 3.09 & $0-21$ & 2.10 & $<0.01$ \\
\hline SAA & 33,446 & $19,242-82,008$ & 51,401 & $21,488-239,797$ & 1.54 & $<0.01$ \\
\hline IL-23p40 & 0 & $0-4.3$ & 1.35 & $0-18.2$ & NA & $<0.0001$ \\
\hline $\mathrm{IL}-1 \alpha$ & 0 & $0-0.6$ & 0.49 & $0-3.7$ & NA & $<0.0001$ \\
\hline IL-2 & 0 & $0-1.3$ & 0.00 & $0-0.2$ & NA & $<0.05$ \\
\hline IL-5 & 0 & $0-0.6$ & 0.26 & $0-2.0$ & NA & $<0.001$ \\
\hline CCL3 & 0 & $0-18$ & 70.3 & $0-755$ & NA & $<0.0001$ \\
\hline TNF- $\alpha$ & 0 & $0-0$ & 0.00 & $0-1.4$ & NA & $<0.01$ \\
\hline \multicolumn{7}{|c|}{ Not significantly different } \\
\hline Flt-1 & 932 & $57-3910$ & 1436 & $471-4431$ & 1.54 & 0.06 \\
\hline VCAM-1 & 3748 & $1647-19,192$ & 4964 & $1134-82,268$ & 1.32 & 0.09 \\
\hline CRP & 87,863 & $45,144-419,932$ & 111,126 & $42,185-298,708$ & 1.26 & 0.33 \\
\hline IL-17A & 0 & $0-7.1$ & 0 & $0-4.2$ & NA & 0.36 \\
\hline IL-7 & 0 & $0-0.6$ & 0 & $0-1.3$ & NA & 0.09 \\
\hline TNF- $\beta$ & 0 & $0-0$ & 0 & $0-1.1$ & NA & 0.49 \\
\hline VEGF-C & 0 & $0-26$ & 0 & $0-36.6$ & NA & 0.51 \\
\hline \multicolumn{7}{|c|}{ Significantly reduced } \\
\hline Tie-2 & 2849 & $149-4208$ & 1712 & $125-4280$ & 0.60 & $<0.001$ \\
\hline FGF2 & 9734 & $258-17,546$ & 3141 & $408-11,371$ & 0.32 & $<0.0001$ \\
\hline VEGF-D & 76.7 & $0-237$ & 24.3 & $0-193$ & 0.32 & $<0.0001$ \\
\hline
\end{tabular}

CRP, C-reactive protein; FGF, fibroblast growth factor; Flt, Fms-like tyrosine kinase 1; GM-CSF, granulocyte-macrophage colony-stimulating factor; ICAM, intercellular adhesion molecule; IFN, interferon; IP-10, interferon gamma-induced protein 10; NA, not applicable; PIGF, placental growth factor; SAA, serum amyloid A; Tie, tyrosine kinase; TKA, total knee arthroplasty; TNF, tumor necrosis factor; VCAM, vascular cell adhesion molecule; VEGF, vascular endothelial growth factor.

10 -fold cross validation to prevent overfitting. Data were weighted so that each class (primary or revision) had equal weight, despite different numbers of observations. In using the single marker CCL2 alone to classify primary/revision TKA patients, the model was fitted using the base $\mathrm{R}$ glm function and the receiver operating characteristic curve produced with the R package pROC. ${ }^{34}$ The median and CIs on the area under the curve were calculated with the ci.auc function.

\section{Statistical Analysis}

Markers in synovial fluid, synovial membrane, and infrapatellar fat pat were grouped into those significantly elevated, those significantly decreased, and those not significantly different or undetectable in patients undergoing primary and revision TKA. Data are presented as a Venn diagram, graphically as change in median expression levels, as box-and-whiskers plots for individual 
Table 4 Synovial Membrane: Primary TKA versus Revision TKA

\begin{tabular}{|c|c|c|c|c|c|c|}
\hline \multirow[b]{2}{*}{ Marker } & \multicolumn{2}{|l|}{ Primary TKA } & \multicolumn{2}{|l|}{ Revision TKA } & \multicolumn{2}{|c|}{ Revision versus Primary TKA } \\
\hline & Median, $\mathrm{pg} / \mathrm{mL}$ & Range, $\mathrm{pg} / \mathrm{mL}$ & Median, pg/mL & Range, $\mathrm{pg} / \mathrm{mL}$ & Fold change & $P$ value \\
\hline \multicolumn{7}{|c|}{ Significantly increased } \\
\hline CCL3 & 4.4 & $0-24$ & 51.7 & $0-750$ & 11.74 & $<0.0001$ \\
\hline CCL4 & 6.0 & $0-65$ & 33.0 & $0-380$ & 5.53 & $<0.01$ \\
\hline IP-10 & 13.3 & $3-1183$ & 44.7 & $3-2507$ & 3.37 & $<0.01$ \\
\hline CCL17 & 0.99 & $0-58$ & 1.68 & $0-19$ & 1.69 & $<0.05$ \\
\hline IL-23p40 & 0 & $0-7.1$ & 1.08 & $0-10.8$ & NA & $<0.001$ \\
\hline IL-13 & 0 & $0-3.5$ & 0.98 & $0-5.1$ & NA & $<0.01$ \\
\hline TNF- $\alpha$ & 0 & $0-1.4$ & 0.00 & $0-3.3$ & NA & $<0.05$ \\
\hline \multicolumn{7}{|c|}{ Not significantly different } \\
\hline VCAM-1 & 3799 & $1420-25,228$ & 5163 & $2311-45,561$ & 1.36 & 0.09 \\
\hline VEGF & 10.3 & $0-46$ & 13.8 & $0-362$ & 1.33 & 0.94 \\
\hline IL-16 & 382 & $147-1743$ & 493 & $135-3003$ & 1.29 & 0.21 \\
\hline ICAM-1 & 33,978 & $15,606-115,625$ & 36,721 & $17,683-95,444$ & 1.08 & 0.25 \\
\hline SAA & 41,586 & $22,888-84,910$ & 43,694 & $21,989-159,994$ & 1.05 & 0.21 \\
\hline PIGF & 39.4 & $11-353$ & 39.8 & $11-116$ & 1.01 & 0.81 \\
\hline CCL11 & 6.61 & $0-34$ & 6.53 & $0-41$ & 0.99 & 0.36 \\
\hline CCL13 & 75.0 & $17-1199$ & 70.7 & $6-609$ & 0.94 & 0.80 \\
\hline Flt-1 & 1727 & $646-7201$ & 1466 & $526-5691$ & 0.85 & 0.32 \\
\hline IL-4 & 0.04 & $0-0.1$ & 0.03 & $0-0.2$ & 0.81 & 0.90 \\
\hline GM-CSF & 0 & $0-1.4$ & 0 & $0-1.1$ & NA & $>0.99$ \\
\hline TNF- $\beta$ & 0 & $0-1.7$ & 0 & $0-1.7$ & NA & 0.64 \\
\hline VEGF-C & 0 & $0-114$ & 0 & $0-30$ & NA & 0.60 \\
\hline \multicolumn{7}{|c|}{ Significantly reduced } \\
\hline Tie-2 & 3364 & $2006-4518$ & 2322 & $138-5181$ & 0.69 & $<0.001$ \\
\hline CCL26 & 14.4 & $4-104$ & 8.7 & $0-55$ & 0.61 & $<0.01$ \\
\hline CRP & 255,593 & $46,461-1,128,993$ & 121,677 & $42,048-355,751$ & 0.48 & $<0.01$ \\
\hline FGF2 & 8779 & $3453-15,081$ & 3781 & $679-13,018$ & 0.43 & $<0.0001$ \\
\hline VEGF-D & 66.5 & $8-390$ & 22.7 & $0-249$ & 0.34 & $<0.05$ \\
\hline
\end{tabular}

CRP, C-reactive protein; FGF, fibroblast growth factor; Flt, Fms-like tyrosine kinase 1; GM-CSF, granulocyte-macrophage colony-stimulating factor; ICAM, intercellular adhesion molecule; IFN, interferon; IP-10, interferon gamma-induced protein 10; NA, not applicable; PIGF, placental growth factor; SAA, serum amyloid A; Tie, tyrosine kinase; TKA, total knee arthroplasty; TNF, tumor necrosis factor; VCAM, vascular cell adhesion molecule; VEGF, vascular endothelial growth factor.

markers, and in tabular form. Significance was calculated using $U$-test. Correlations between IL- $1 \alpha$ levels and CCL2, IL-6, and IL-8 levels were tested using linear regression. In vitro data are presented as means \pm SEM. Differences between treatment groups were analyzed using paired $t$-test (Graphpad Prism 6; GraphPad Software Inc., La Jolla, CA). Significance was set at $P<0.05$.

\section{Results}

\section{Chronic Inflammation Is a Common Feature of the Postsurgical Joint}

The post-TKA joint is associated with the persistence of fibroblasts and extensive fibrotic remodeling of the replacement joint (Figure 1A). ${ }^{22,35}$ To determine the degree to which TKA 




B


Figure 2 Markers of inflammation are elevated in patients undergoing revision total knee arthroplasty (TKA). Fold change in protein expression in synovial fluid (A), infrapatellar fat pad (B), and synovial membrane (C) isolated from patients undergoing revision TKA compared with patients undergoing primary TKA. Fold change was calculated using median protein expression. Significance was calculated using $U$-test. ${ }^{*} P<0.05,{ }^{* *} P<0.01,{ }^{* * *} P<0.001$, and $* * * * P<0.0001$ primary versus revision patients. CRP, C-reactive protein; FGF, fibroblast growth factor; Flt, Fms-like tyrosine kinase 1; GM-CSF, granulocytemacrophage colony-stimulating factor; ICAM, intercellular adhesion molecule; IFN, interferon; IP-10, interferon gamma-induced protein 10; PIGF, placental growth factor; SAA, serum amyloid A; Tie, tyrosine kinase; TNF, tumor necrosis factor; VCAM, vascular cell adhesion molecule; VEGF, vascular endothelial growth factor.

is associated with inflammation, synovial fluid, synovial membrane, and infrapatellar fat pad were collected from patients undergoing primary or revision surgery and the expression of 39 soluble inflammatory proteins was profiled at each anatomical location. When comparing protein expression between pre- and post-TKA samples, it was discovered that all three anatomical locations in the fibrotic joint display an enhanced proinflammatory state (Tables 2, 3, and 4, Figure 2, and Supplemental Figures S1, S2, and S3). Synovial fluid was the most dramatically affected, with elevated expression of 26 inflammatory markers, followed by the infrapatellar fat pad (22 up-regulated proteins) and the synovial membrane (10 upregulated proteins). These data were used to construct a Venn diagram (Figure 1B) illustrating the anatomical location of the up-regulated inflammatory markers. CCL2, CCL3 (MIP-1 $\alpha$ ), CCL4 (MIP-1 $\beta$ ), CCL17 (TARC), CCL22 (MDC), 
IL-8, IP-10, IL-23p40, and tumor necrosis factor- $\alpha$ were upregulated in all three regions of the fibrotic joint. By far the most induced protein was IL-8, which was expressed at approximately 35 -fold, approximately 40 -fold, and approximately 80-fold higher levels in the synovial fluid, infrapatellar fat pad, and synovial membrane, respectively (Figure 2). The synovial fluid was the most proinflammatory, with eight proteins exclusively elevated in revision TKA compared with primary TKA. These proteins were the granulocyte recruitment ligands CCL13 (monocyte chemoattractant protein-4) and CCL26 (eotaxin-3), the granulocyte modulator granulocyte-macrophage colony-stimulating factor, tumor necrosis factor- $\beta$, the cleaved form of vascular cell adhesion molecule 1, and proangiogenic factors vascular endothelial growth factor-C and Fms-like tyrosine kinase 1 (Figure 1B). Together, these markers suggest the synovial fluid of the fibrotic joint resembled a state of chronic allergic inflammation. ${ }^{36}$ Up-regulated T-cell stimulators IL-2, IL-15, and IL-16, the inflammatory cytokines IL- $1 \alpha$, IL- $1 \beta$, and IL-5, and the cleaved form of intercellular adhesion molecule 1 were common to the synovial fluid and infrapatellar fat pad of the fibrotic joint.

\section{Fibroblasts from the Infrapatellar Fat Pad Polarize to a Proinflammatory Phenotype in Response to IL- $1 \alpha$}

Having established that the fibrotic post-TKA joint acquires a state of unresolved inflammation, it was next determined if fibroblasts contribute to this state. We previously reported that the infrapatellar fat pad is a site at which extensive fibrotic remodeling and fibroblast accumulation occur after TKA. ${ }^{22}$ Furthermore, we now demonstrate that fibroblasts in the postTKA joint express IL-1R1 (Figure 1A). IL-1 $\alpha$ polarizes fibroblasts toward a proinflammatory state ${ }^{19}$ and is elevated in both the post-TKA infrapatellar fat pad and synovial fluid (Figure 1B). Fibroblasts isolated from the infrapatellar fat pad and exposed to $\mathrm{pg} / \mathrm{mL}$ levels of IL- $1 \alpha$ underwent a dramatic induction of CCL2, IL-6, and IL-8, all being secreted at $\mathrm{ng} / \mathrm{mL}$ levels. By contrast, profibrogenic TGF- $\beta 1$ failed to induce this proinflammatory state (Figure 3A). IL-6 was remarkable in that it was a unique inflammatory feature of the infrapatellar fat pad in the post-TKA joint (Figure 1B). Multiarray protein measurements confirmed that IL-6 is up-regulated in the remodeled infrapatellar fat pad, but remains unchanged in the synovial fluid (Figure 3, B and C). Moreover, elevated IL-1 $\alpha$ in the fibrotic infrapatellar fat pad (pg/mL levels) correlated with enhanced expression of CCL2, IL-6, and IL-8 (Figure 3D), suggesting that fibroblasts exposed to IL- $1 \alpha$ in the microenvironment of the fibrotic infrapatellar fat pad are likely to be a major source of proinflammatory CCL2, IL-6, and IL-8. In agreement with this, immunocytochemistry performed on the post-TKA infrapatellar fat pad identified a significant number of the vimentinpositive fibroblasts that were also positive for IL-6 (Figure 1C).

To determine intracellular signaling pathways responsible for mediating IL- $1 \alpha$-induced polarization of fibroblasts, the cells were treated with inhibitors of downstream signal transduction molecules. Inhibitors of Janus activating kinase 2 , transforming growth factor $\beta$-activated kinase 1 (TAK1), and IKK-2, signaling molecules known to activate the cardinal proinflammatory transcription factor $\mathrm{NF}-\kappa \mathrm{B},{ }^{37-39}$ blunted IL- $1 \alpha$-induced expression of CCL2 by twofold or greater (Figure 3, E and F, and Supplemental Figure S4).

To investigate the in vivo relevance of these data, a systems bioinformatics analysis was performed using the protein expression data derived from the multiarray protein measurements of up-regulated proinflammatory molecules in synovial fluid of the post-TKA joint (Table 2 and Figure 1B). By using a network reconstruction technique, the innate immune protein interactome of the remodeled TKA joint was interrogated (Figure 4). Of particular note was the mapping of NF- $\kappa B$ family proteins Rel (c-Rel), RelA (p65), RelB, and NF-кB1 (p50) in a network including CCL17, IP-10, CCL2, CCL22, CCL3, CCL4, CCL11 (eotaxin), and IL-15, all of which were identified as up-regulated in one or both of the synovial fluid and infrapatellar fat pad of the fibrotic post-TKA joint (Figure 4A). The reconstructed network also contained TAK1, IKK-2, and the Janus activating kinase 2 target STAT3, rationalizing their role in the CCL2 response to IL- $1 \alpha$. Proteins in the network and in highly connected submodules were also enriched for gene ontology processes, including leukocyte migration and response to wound healing (Figure 4B).

\section{Inflammatory Fibroblasts Regulate Monocyte Recruitment via NF- $\kappa \mathrm{B}-$ Regulated Expression of CCL2}

Having established that IL- $1 \alpha$ polarizes fibroblasts toward an inflammatory phenotype, it was next tested if these cells are able to directly stimulate recruitment of immune cells, specifically monocytes. To test this hypothesis, a transwell migration assay was performed with human THP-1 monocytes and conditioned media from unstimulated, IL- $1 \alpha$-stimulated, or TGF- $\beta 1-$ stimulated fibroblasts. Conditioned media from IL- $1 \alpha$-stimulated fibroblasts significantly increased migration of monocytes compared with unstimulated cells. TGF- $\beta 1$ treatment had no effect on monocyte migration (Figure 5A). Pretreating the conditioned media with neutralizing antibodies against CCL2 and IL-6 identified CCL2 as the predominant mediator of monocyte recruitment, such that anti-CCL2 reduced monocyte recruitment by approximately $80 \%$; in contrast, anti-IL-6 had negligible effects (Figure 5B). Treatment of IL- $1 \alpha$-stimulated fibroblasts with inhibitors of Janus activating kinase 2 , TAK1, and IKK effectively prevented their ability to recruit monocytes, confirming the requirement of NF- $\kappa$ B activation (Figure 5C). Because CCL2 was found to be significantly elevated in the post-TKA synovial fluid and infrapatellar fat pad and is integrated with NF- $\kappa \mathrm{B}$ in the innate immune interactome of the fibrotic joint, $\mathrm{CD} 68^{+}$ monocytes in the fibrotic joint were quantified. There was a significant increase in the number of $\mathrm{CD} 68^{+}$monocytes in the infrapatellar fat pad of fibrotic revision post-TKA joints compared with nonfibrotic primary TKA joints (Figure 5D). 
A

CCL2


B

\section{Synovial fluid}


C
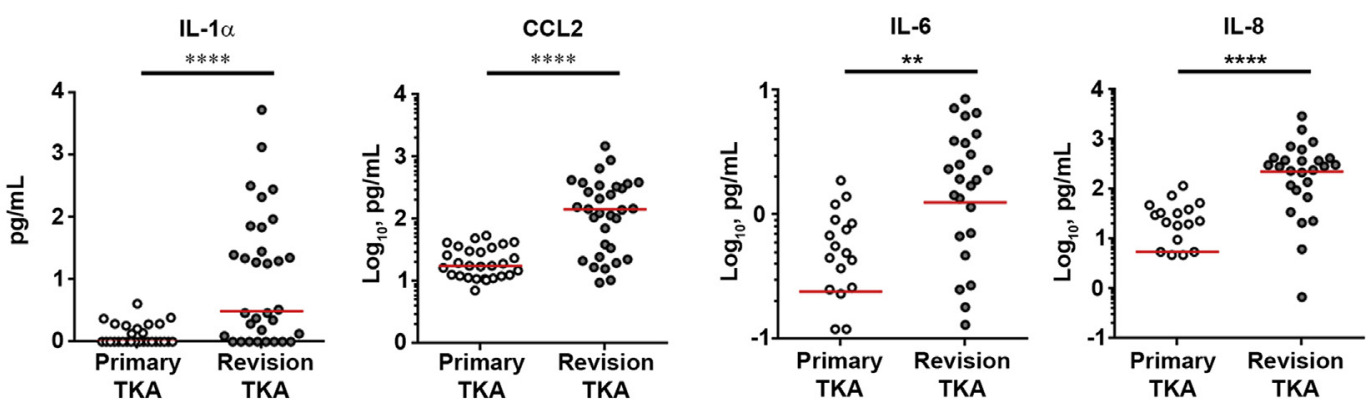

D


E

Fibroblasts from primary TKA

$\mathbf{F}$

Fibroblasts from revision TKA
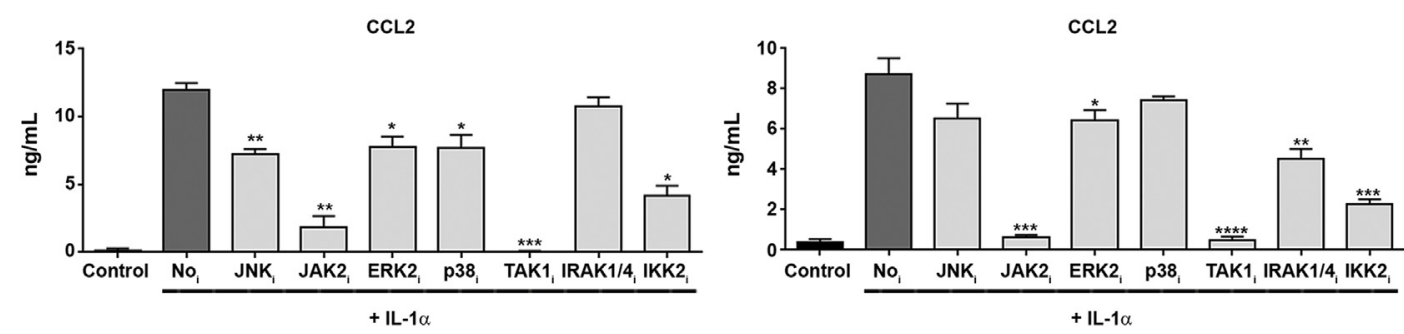
Elevated CCL2 in the Inflammatory Environment of the Fibrotic Post-TKA Joint Correlates with Pain

The degree to which the inflammatory profile of the synovial fluid of the joint stratifies patients into those requiring revision TKA surgery was quantified. Inflammatory proteins with the greatest fold change between primary and revision patients were log transformed and used for hierarchical clustering, which was visualized by a heat map. The use of inflammatory protein expression in the synovial fluid was sufficient to segregate revision from primary TKA patients with only $n=1$ revision and $n=2$ primary TKA patients misclassified (Figure 6A). The predictive value of the inflammatory profile of the infrapatellar fat pad (Supplemental Figure S5A) and the synovial membrane (Supplemental Figure S6A) was also assessed. Although a degree of stratification was observed with these tissues, there was a larger degree of overlap between the patient groups compared with synovial fluid. Principal component analysis was performed to further investigate the predictive value of the inflammatory profile in the synovial fluid (Figure 6B). This approach demonstrated the excellent predictive value of the synovial fluid, with distinct separation of primary and revision TKA samples (only $n=1$ revision TKA patient clustering incorrectly). Similar analysis of the infrapatellar fat pad (Supplemental Figure S5B) and synovial membrane (Supplemental Figure S6B) again demonstrated a much larger degree of overlap compared with synovial fluid. The $\mathrm{R}$ package glmnet was used to select a reduced set of inflammatory markers that reliably classify primary and revision TKA patients. Again, the synovial fluid had the most predictive value and indicated that a single inflammatory marker, CCL2, achieved a misclassification error of $<5 \%$ (Figure 6C). Indeed, using CCL2 alone to generate a receiver operating characteristic curve achieved an area under the curve of $96.8 \%$ (95\% CI, 89\%-100\%) in stratifying patients as primary or revision TKA (Figure 6D). The misclassification error was significantly higher for the infrapatellar fat pad (Supplemental Figure S5C) and synovial membrane (Supplemental Figure S6C), whereas the ability of CCL2 alone to stratify patients was also reduced (infrapatellar fat pad: area under the curve, $84.2 \%$; 95\% CI, 74\%-95\%) (Supplemental Figure S5D) (synovial membrane: area under the curve, $76.9 \%$; 95\% CI, 65\%-89\%) (Supplemental Figure S6D). For these tissues, glmnet analysis suggested that more complex models containing additional markers, such as CCL3, CCL13, CCL22, IL-8, and C-reactive protein, may be better able to stratify patients, but would still not match the accuracy of CCL2 in synovial fluid (Supplemental Figures S5C and S6C).

Because degree of pain and stiffness are clinical measures of success of the post-TKA joint and patient quality of life, ${ }^{25}$ the degree to which these clinical outcomes correlated with inflammatory markers in the joint was determined. Western Ontario and McMaster Universities Osteoarthritis Index (WOMAC) scores for pain and stiffness were obtained from the Newcastle Freeman Joint Registry for patients consented to this study and correlated to levels of CCL2 and other markers significantly elevated in post-TKA patients. A significant correlation between CCL2 levels in synovial fluid and WOMAC pain scores was observed, but no correlation with WOMAC stiffness scores was found (Figure 7). A significant positive correlation was also seen with vascular endothelial growth factor-C and other markers elevated after TKA, such as IL-8, CCL22, CCL3, and tumor necrosis factor- $\alpha$; these markers also demonstrated a nonstatistically significant correlation with WOMAC pain scores. Again, there was little to no correlation between any of the markers and WOMAC stiffness scores. Interestingly, levels of CCL2, IL-8, CCL22, and CCL3 in the infrapatellar fat pad were significantly correlated with WOMAC stiffness scores but not with WOMAC pain scores (Supplemental Figure S7). Levels of marker expression did not significantly correlate with either WOMAC pain or stiffness scores in synovial membrane (Supplemental Figure S8).

We conclude that inflammatory markers in the post-TKA synovial fluid correlate with pain and that CCL2, a factor produced by inflammatory fibroblasts in the fibrotic joint, is sufficient to predict pain and identify patients requiring revision TKA surgery.

\section{Discussion}

Modern lower limb arthroplasty results in pain relief and satisfactory outcomes in $>80 \%$ of patients. However, as many as $20 \%$ of patients express post-surgical dissatisfaction because of persistent pain, swelling, loss of movement, and reduced functional ability in the absence of joint

Figure 3 Markers elevated after total knee arthroplasty (TKA) are associated with fibroblast activation. A: Fibroblasts isolated from the infrapatellar fat pad of patients undergoing primary TKA were treated with control media, IL- $1 \alpha(500 \mathrm{pg} / \mathrm{mL})$, or transforming growth factor (TGF)- $\beta 1(3 \mathrm{ng} / \mathrm{mL})$ for $24 \mathrm{hours}$ and secreted levels of CCL2, IL-6, and IL-8, determined by enzyme-linked immunosorbent assay (ELISA). Data are analyzed using paired $t$-tests. B and C: IL-1 $\alpha$, CCL2, IL-6, and IL-8 protein levels were quantified in synovial fluid (B) and infrapatellar fat pad (C) and compared between patients undergoing primary and revision TKA. Data are presented as individual patient values, with median signified by the red line and analyzed using a $U$-test. D: IL-1 $\alpha$ protein expression correlates against CCL2, IL-6, and IL-8 levels in synovial fluid. Data are presented as individual patient values with associated linear regression analysis. E and F: Fibroblasts isolated from the infrapatellar fat pad of patients undergoing primary (E) and revision (F) TKA were pretreated for 1 hour with several selective inhibitors [TAK1 inhibitor $\left(\mathrm{TAK}_{\mathrm{i}}\right), 1 \mu \mathrm{mol} / \mathrm{L}$; all other inhibitors, $10 \mu \mathrm{mol} / \mathrm{L}$ ] before stimulation with IL- $1 \alpha(500 \mathrm{pg} / \mathrm{mL})$ for 24 hours, and CCL2 was quantified by ELISA. Data are analyzed using paired $t$-tests. Data are presented as means $\pm \operatorname{SEM}(\mathbf{A}, \mathbf{E}$, and $\mathbf{F}) . n=6(\mathbf{A}, \mathbf{E}$, and $\mathbf{F}) .{ }^{*} P<0.05,{ }^{* *} P<0.01, * * * P<0.001$, and ${ }^{* * * *} P<0.0001$ primary versus revision patients. ERK, extracellular signal-regulated kinase; IKK, IK B kinase; IRAK, IL-1 receptor associated kinase; JAK, Janus activating kinase; JNK, C-Jun N-terminal kinase; MCP, monocyte chemoattractant protein. 


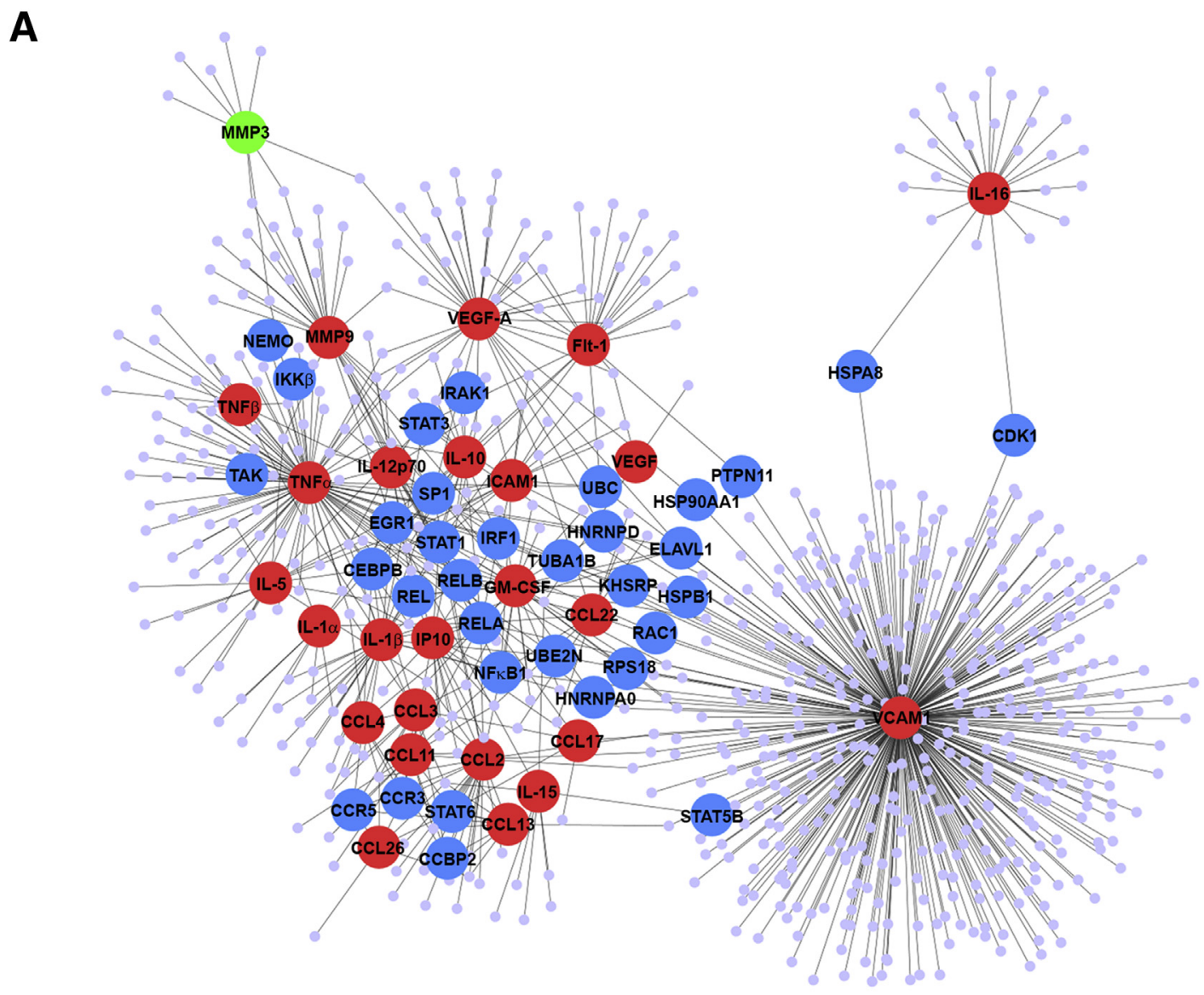

B



Leukocyte migration

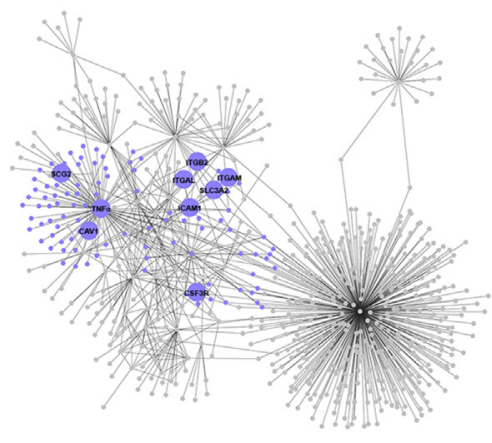

Response to wound healing



Figure 4 Network and module analysis. A: Protein-protein interaction network built from differentially expressed markers. Red indicates up-regulated marker protein; green, down-regulated marker protein; blue, proteins with either degree (number of interactions) $\geq 5$, betweenness centrality $\geq 0.01$, or one of a selected set of proteins of interest [transforming growth factor $\beta$-activated kinase, IL-1 receptor associated kinase (IRAK) 1 , STAT3, IK B kinase (IKK) $\beta$, or NF-kappa-B essential modulator]. B: Network with one module (highly connected subnetwork) highlighted in purple. Large labeled nodes show proteins associated with selected enriched gene ontology biological processes mapped to the module; highlighted nodes are associated with the processes positive regulation of NF- $\kappa B$ transcription factor activity, leukocyte migration, and response to wound healing. CCBP, chemokine-binding protein 2; CEBPB, CCAAT/ enhancer binding protein beta; EGR, early growth response protein 1; GM-CSF, granulocyte-macrophage colony-stimulating factor; HSP, heat shock protein; MMP, matrix metalloproteinase; TNF, tumor necrosis factor; VEGF, vascular endothelial growth factor.

instability or infection. ${ }^{25}$ The epidemiology and etiology of this postoperative state remain poorly understood, in particular with respect to pain, yet the symptoms are often sufficiently severe to necessitate early revision surgery, within 5 years of the primary operation. The aim of this study was to determine the nature of the inflammatory process occurring in joints requiring revision surgery and to interrogate the potential role of the fibroblast to contribute to stress-induced inflammatory exacerbations and pain in the post-TKA knee. 
A

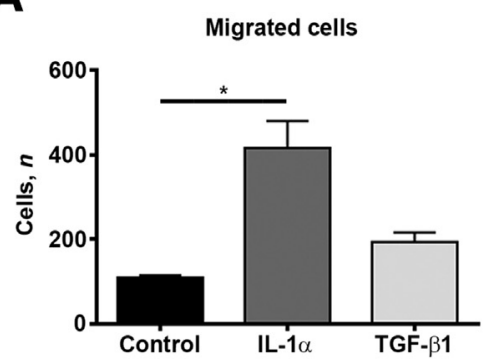

B

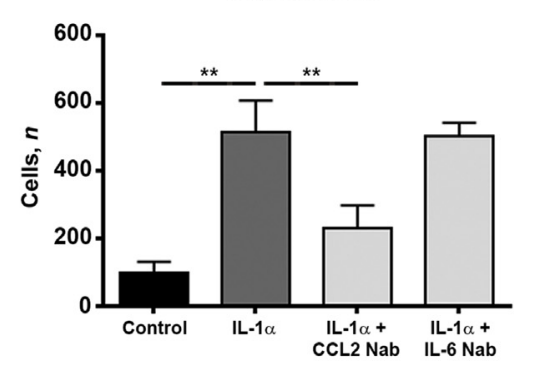

C



D



Extensive fibrotic remodeling is found to be common to revised TKA joints and is characterized by the accumulation of fibrotic extracellular matrix into the infrapatellar fat pad and synovial membrane. ${ }^{22}$ The fibrotic matrix of the TKA joint is also characterized by the persistence of high numbers of $\alpha$-smooth muscle actin - and IL-1R1-positive fibroblasts. ${ }^{19,35}$ Herein, we discovered that the fibrotic postTKA knee is characterized by a chronic inflammatory signature and identify IL-1R1-positive fibroblasts as a potential source of proinflammatory mediators that promote monocyte recruitment and pain in the TKA joint. This occurs via a paracrine pathway in which soluble IL- $1 \alpha$ stimulates NF- $\kappa \mathrm{B}-$ dependent expression of CCL2. This illuminates a new disease model in which nonresolving fibrosis becomes a driver of inflammation in response to the sterile innate immune triggers (IL-1 $\alpha$ ). This model has potential relevance to the pathophysiology of all active fibrotic tissues and may help explain the poorly understood association of fibrosis with inflammatory flares and chronic pain in osteoarthritic (OA) joints. We further propose that targeting the fibroblast in the setting of the fibrotic joint offers a novel and rationale approach for suppressing inflammation and pain (ie, worthy of further investigation).
Figure 5 IL-1 $\alpha$-activated fibroblasts promote recruitment of monocytes by secreting CCL2. Fibroblasts isolated from the infrapatellar fat pad of patients undergoing primary total knee arthroplasty (TKA) were stimulated with control media, IL- $1 \alpha(500 \mathrm{pg} / \mathrm{mL})$, or transforming growth factor (TGF)- $\beta 1 \quad(3 \mathrm{ng} / \mathrm{mL})$ for 24 hours to generate conditioned media for transwell migration assays. A: Number of monocytes recruited by conditioned media from unstimulated (control), IL- $1 \alpha-$ stimulated, and TGF- $\beta 1-$ stimulated fibroblasts. B: Conditioned medium from IL- $1 \alpha$-stimulated fibroblasts was pretreated for 1 hour with neutralizing antibodies against CCL2 and IL- 6 and then used in transwell migration assays. C: Conditioned medium from fibroblasts pretreated for 1 hour with several selective inhibitors (transforming growth factor $\beta$-activated kinase, $1 \mu \mathrm{mol} / \mathrm{L}$; all other inhibitors, $10 \mu \mathrm{mol} / \mathrm{L}$ ) before stimulation with IL$1 \alpha$ for 24 hours and then used in transwell migration assays. The number of migrated cells was quantified on a Nikon Eclipse microscope. Data are analyzed using paired $t$-tests. D: Number of $\mathrm{CD} 8^{+}$cells in the infrapatellar fat pad of patients undergoing primary and revision TKA was quantified in $10 \times$ randomly selected high-powered fields using Nikon NIS elements image analysis software. Data are analyzed using $U$-test. Data are presented as means $\pm \mathrm{SEM}$ (C and D). $n=6(\mathbf{A}-\mathbf{C})$. ${ }^{*} P<0.05,{ }^{* *} P<0.01$ versus $\mathrm{No}_{i}$. Original magnification, $\times 20$ (D). ERK, extracellular signal -regulated kinase; IKK, Iא B kinase; IRAK, IL-1 receptor associated kinase; JAK, Janus activating kinase; JNK, c-Jun $\mathrm{N}$-terminal kinase.
Our key findings are as follows: i) the synovial fluid and infrapatellar fat pad of painful TKA joints displayed elevated expression of multiple inflammatory proteins, including IL-1 $\alpha$ and CCL2; ii) bioinformatics analyses of soluble protein expression in the inflamed joint indicated convergence on $\mathrm{NF}-\kappa \mathrm{B}$ signaling, indicative of a state of chronic unresolved inflammation; iii) fibroblasts in the fibrotic infrapatellar fat pad express IL-1R1 and IL-6 in vivo and in response to IL- $1 \alpha$, polarized toward a highly inflammatory phenotype characterized by induction of CCL2 in vitro; and iv) CCL2 levels in the joint closely correlated with the degree of pain. From a translational perspective, the main strength of this study is that it identified the association of IL- $1 \alpha / \mathrm{NF}-\kappa \mathrm{B} / \mathrm{CCL} 2$ inflammatory signaling in fibroblasts as a driver of inflammation and pain using directly relevant human TKA tissues. Furthermore, this novel pathway offers multiple points for therapeutic intervention using drugs that are safe and clinically effective in humans. A limitation of the study is the limited information available on the revision patients referred into our unit for specialist treatment. Specifically, we do not know whether all of the revision patients had their infrapatellar fat pad excised during their primary surgery and the extent of the synovial resection initially 


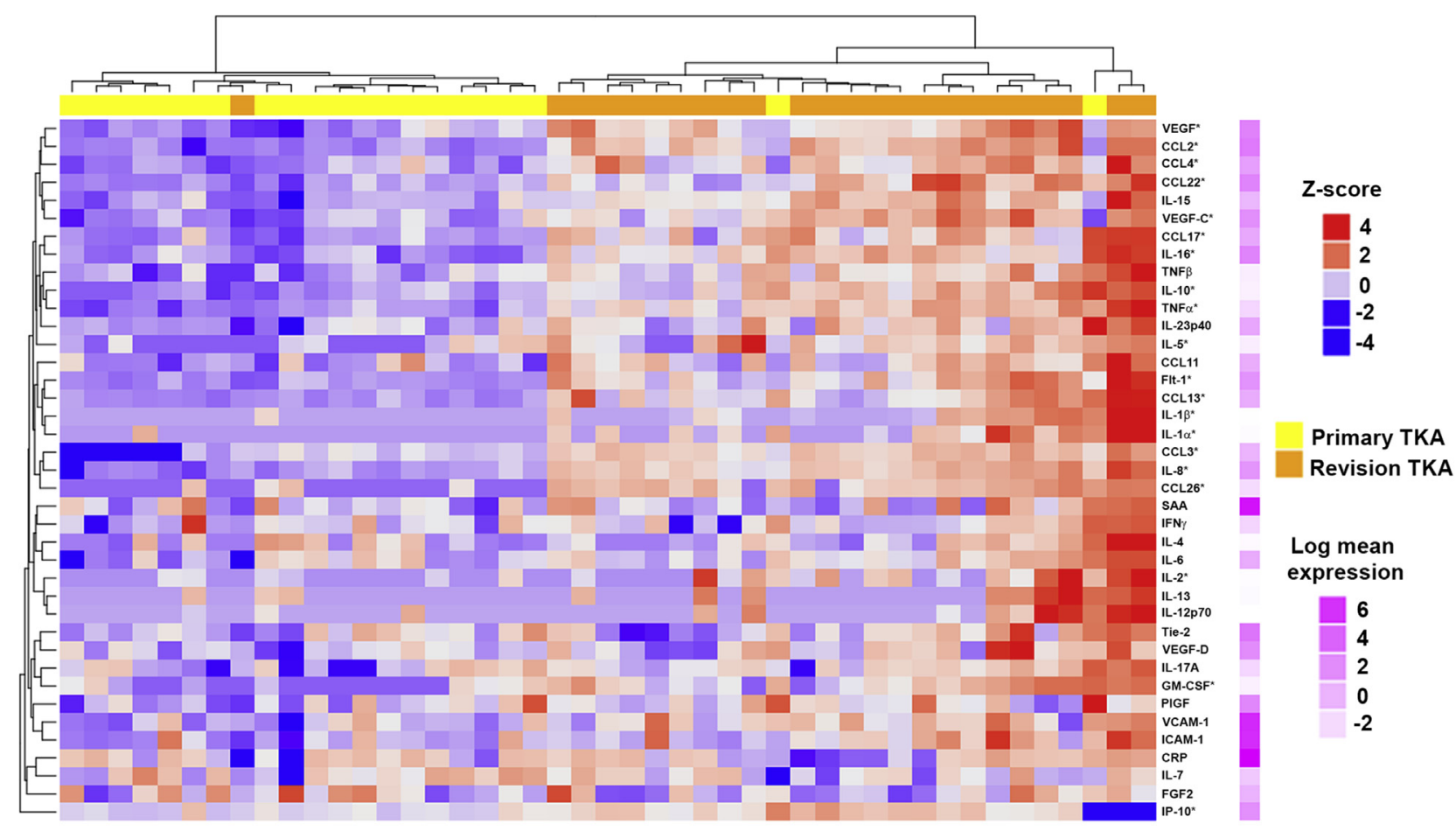

B

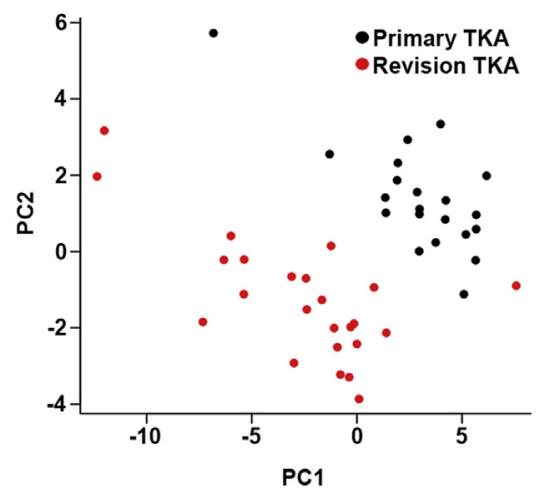

C



D

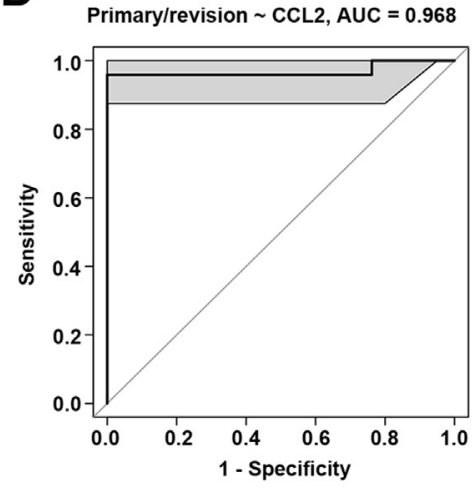

Figure 6 CCL2 in synovial fluid can be used to stratify patients undergoing revision total knee arthroplasty (TKA) from patients undergoing primary TKA. A: Heat map of log-transformed protein intensity in synovial fluid isolated from patients undergoing primary or revision TKA clustered using the differentially expressed markers with the highest fold change. Asterisks indicate markers that were used for clustering analysis/stratification. B: Principal component analysis plot of all patients using all markers. C: Classification error as function of the number of markers included, cross validated using glmnet package. Dotted lines mark two important values of the regularization parameter $\lambda$, which controls the number of markers included. On the left is the value of $\lambda$ that gives the minimum mean cross-validated error. On the right is the value that gives the most regularized model, such that error is within one SEM of the minimum. D: Receiver operating characteristic curve for classification with CCL2 alone [area under the curve (AUC), 0.968; 95\% CI, 0.89-1.0]. CRP, C-reactive protein; FGF, fibroblast growth factor; Flt, Fms-like tyrosine kinase 1; GM-CSF, granulocyte-macrophage colony-stimulating factor; ICAM, intercellular adhesion molecule; IFN, interferon; IP-10, interferon gamma-induced protein 10; PIGF, placental growth factor; SAA, serum amyloid A; Tie, tyrosine kinase; TNF, tumor necrosis factor; VCAM, vascular cell adhesion molecule; VEGF, vascular endothelial growth factor.

performed. Fat pad resection has been associated with increased anterior knee pain and patella baja, which is suggestive of increased intra-articular scarring. ${ }^{40,41}$

Traditionally, fibroblasts are considered as structural cells that, in response to tissue damage, adopt an activated fibroblast state and promote wound healing and fibrogenesis via their secretion of fibrous collagens and inhibitors of collagenases. ${ }^{4}$ Less well recognized is that these cells exhibit substantial heterogeneity and phenotypic plasticity and can adopt fibroblastic, contractile, or inflammatory states under the influence of cues in their microenvironment. ${ }^{13}$ Polarization of fibroblasts to an inflammatory state is characterized by their acquired expression of IL-6 and IL-8. Studies in fibroblasts derived 


\section{Synovial fluid}
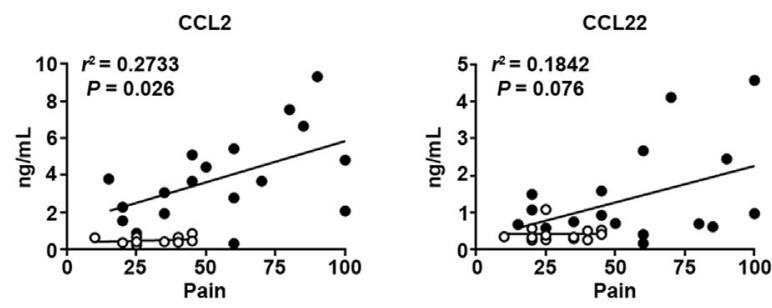

CCL3

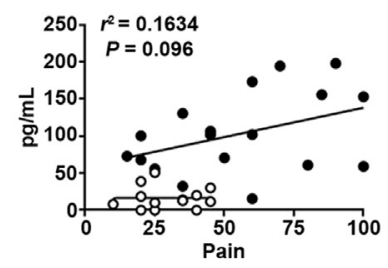

VEGF-C

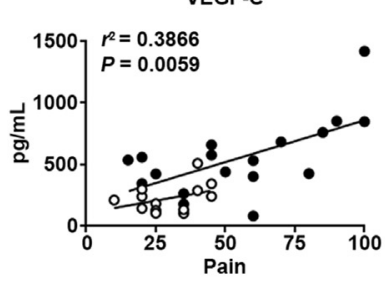

IL-6

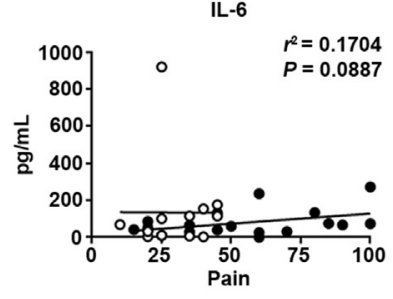

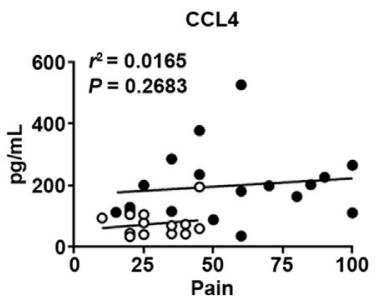

TNF- $\alpha$

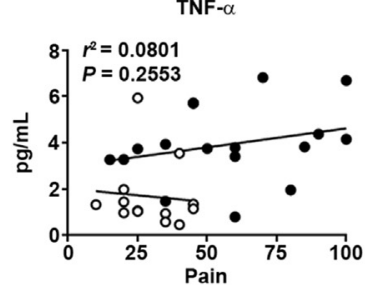

IL-8



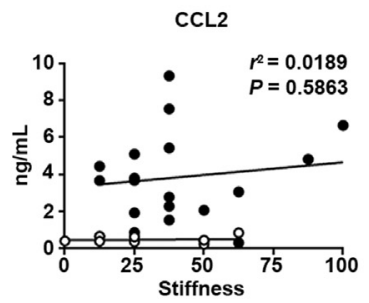
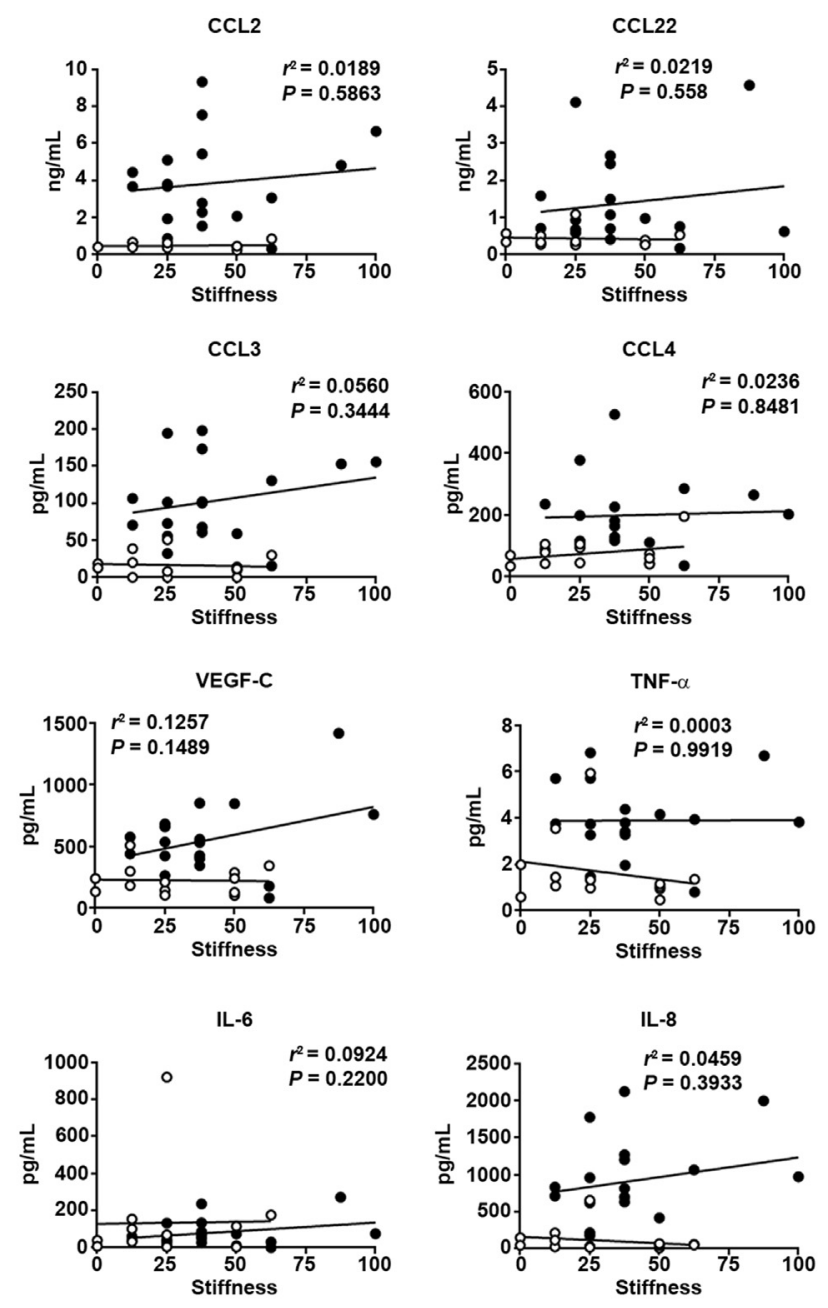

O Primary TKA • Revision TKA

Figure 7 Pain positively correlates with levels of CCL2 in synovial fluid. Pain and stiffness, as determined by Western Ontario and McMaster Universities 0steoarthritis Index scores, were plotted against expression levels of several markers that were significantly elevated in the synovial fluid of patients undergoing revision total knee arthroplasty (TKA). Data are presented for patients undergoing primary and revision TKA, and correlations were determined by linear regression analysis. Increased levels of CCL2 are highly predictive of pain but are poorly correlated to stiffness. $n=13$ primary TKA group; $n=18$ revision TKA group. TNF, tumor necrosis factor; VEGF, vascular endothelial growth factor.

from multiple tissues (eg, lung, intestine, heart, and skin) indicate that the innate immune trigger IL- $1 \alpha$ is a powerful paracrine stimulator of an inflammatory polarization of their phenotype. ${ }^{12,14,15,17,42}$ It was found to be true for fibroblasts derived from the infrapatellar fat pad, such that exposure to $\mathrm{pg} / \mathrm{mL}$ levels of IL- $1 \alpha$ induced $\mathrm{ng} / \mathrm{mL}$ levels of secreted IL-6, IL-8, and CCL2. More important, IL-1R1 and IL-6 expression was located primarily to fibroblasts in the fibrotic infrapatellar fat pad, whereas soluble IL- $1 \alpha$, IL-6, IL-8, and CCL2 were also elevated and correlated with IL-1 $\alpha$ levels in the infrapatellar fat pad of the TKA joint. An important future question will be to identify the cellular source of IL-1 $\alpha$ in the joint because this information will reveal the underlying cellular damage/stress mechanisms that trigger the activation of inflammatory fibroblasts of fibrotic tissue. Potential resident sources of IL$1 \alpha$ in the knee include macrophage-like type $A$ and fibroblast-like type B synovial cells. ${ }^{43,44}$

Fibroblasts persisting in the fibrotic TKA joint express IL-1R1, indicating they are primed for polarization to an inflammatory state on exposure to IL-1 $\alpha$. Scarpa et al ${ }^{42}$ reported that fibroblasts isolated from inflammatory bowel disease tissue expressed IL-1R1, as did intestinal subepithelial fibroblasts in the intestine of mice challenged with a low dose of dextran sulfate sodium. More important, after mice had recovered from this mild intestinal injury, a subsequent intrarectal administration of IL- $1 \alpha$ induced severe colitis, whereas mice that had not undergone prior 
dextran sulfate sodium-induced tissue remodeling failed to mount an inflammatory response to IL-1 $\alpha$. The appearance of IL-1R1-positive fibroblasts, therefore, appears to constitute a tissue memory of previous damage that primes the tissue for a robust inflammatory flare when these cells engage IL- $1 \alpha$ in the microenvironment. This concept challenges the traditional simple linear model of fibrogenesis in which inflammation is the driver of fibrosis. Instead, we propose a dynamic positive feed-forward process in which inflammatory fibroblasts sense local tissue damage and, via release of CCL2 and other chemokines, promote the recruitment and activation of innate immune cells to drive inflammation; this process constitutes a circulus vitiosus.

CCL2 classically operates as a chemoattractant for monocytes by binding to its receptor CCR2 ${ }^{45,46}$ CCL2 is highly expressed in the synovial fluid of OA joints and knees after trauma and, as observed herein, is a feature of the inflammatory synovium and infrapatellar fat pad of the fibrotic TKA joint. ${ }^{47-50}$ Although no data directly demonstrating that CCL2 is driving the recruitment of monocytes in vivo have been presented, we hypothesize that the elevated levels of CCL2 in the post-TKA knee are likely one of the factors contributing to the recruitment of $\mathrm{CD}^{+} 8^{+}$cells in a similar manner to what has recently been reported in OA. ${ }^{51}$ An unexpected and novel finding was the close correlation between levels of CCL2 in the synovial fluid of the TKA joint and reported WOMAC pain scores, although no correlation was observed between levels of CCL2 in infrapatellar fat pad and synovial membrane and reported WOMAC pain scores. Although the synovial fluid is unlikely to contain significant numbers of fibroblasts, it is highly likely that fibroblasts present within joint tissues (including the infrapatellar fat pad and synovial membrane as well as other intra-articular or periarticular tissues, such as the joint capsule) are contributing to chronic inflammation in the synovial fluid, as has been previously reported in OA. ${ }^{52}$ In this context, it is intriguing that sensory neurons express CCR2 and, furthermore, CCL2-CCR2 engagement leads to excitation of nociceptive neurons, an event usually associated with the sensation of pain. ${ }^{53-56}$ In a murine model of OA, genetic deletion of either CCL2 or CCR2 delayed the onset of pain-related behavior. ${ }^{57}$ These observations warrant future studies examining the potential for the use of emerging small-molecule inhibitors of CCR2 as therapies for relieving inflammation and intractable pain in the OA and/or post-TKA joint. Alternatively, a similar outcome may be achieved by the local administration of the IL-1R1 antagonist, anakinra; indeed, there is prior clinical evidence that this approach may be beneficial in TKA. ${ }^{58}$ Because NF- $\kappa \mathrm{B}$ signaling appears to be pivotal for IL$1 \alpha$-induced expression of CCL2 by fibroblasts, there may also be an analgesic benefit to be gained from the local administration of small-molecule inhibitors of NF- $\kappa \mathrm{B}$ or its upstream kinase, IKK. ${ }^{59,60}$ Such an approach may also reduce numbers of fibroblasts in the fibrotic tissue because suppression of NF- $\mathrm{BB}$ in these cells is associated with the induction of apoptosis. ${ }^{61}$

In summary, this study identified an important role for fibrosis-associated fibroblasts as sensors of cellular stress/ damage via engagement of surface-expressed IL-1R1 with the alarmin IL-1 $\alpha$, which leads to secretion of CCL2, a wellestablished stimulator of monocyte recruitment and nociceptive pain. We hypothesize that IL-1R1-expressing fibroblasts persist in unresolved fibrotic tissue and prime the tissue for inflammatory episodes in response to local cell stress/damage. Finally, the inflamed post-TKA joint is an important new human disease model with which to further illuminate the complex cross talk between fibrosis, inflammation, and pain.

\section{Acknowledgments}

We thank our colleagues, past and present, for guidance and support; and Sheryl Mitchell, Victoria Hindmarsh, Sarah A. Grant, Julie Norris, Angela Barnes, Sonia Poolan, and Louise Sutcliffe for assistance with patient consent and tissue acquisition.

\section{Supplemental Data}

Supplemental material for this article can be found at https://doi.org/10.1016/j.ajpath.2017.11.007.

\section{References}

1. Wynn TA, Ramalingam TR: Mechanisms of fibrosis: therapeutic translation for fibrotic disease. Nat Med 2012, 18:1028-1040

2. Pujol N, Boisrenoult P, Beaufils P: Post-traumatic knee stiffness: surgical techniques. Orthop Traumatol Surg Res 2015, 101:S179-S186

3. Kim YS, Youn HK, Kim BS, Choi YJ, Koh YG: Arthroscopic evaluation of persistent pain following supramalleolar osteotomy for varus ankle osteoarthritis. Knee Surg Sports Traumatol Arthrosc 2016, 24:1860-1867

4. Darby IA, Zakuan N, Billet F, Desmouliere A: The myofibroblast, a key cell in normal and pathological tissue repair. Cell Mol Life Sci 2016, 73:1145-1157

5. Wells RG, Schwabe RF: Origin and function of myofibroblasts in the liver. Semin Liver Dis 2015, 35:97-106

6. Kramann R, DiRocco DP, Humphreys BD: Understanding the origin, activation and regulation of matrix-producing myofibroblasts for treatment of fibrotic disease. J Pathol 2013, 231:273-289

7. Bonner JC: Mesenchymal cell survival in airway and interstitial pulmonary fibrosis. Fibrogenesis Tissue Repair 2010, 3:15

8. Kisseleva T, Cong M, Paik Y, Scholten D, Jiang C, Benner C, Iwaisako K, Moore-Morris T, Scott B, Tsukamoto H, Evans SM, Dillmann W, Glass CK, Brenner DA: Myofibroblasts revert to an inactive phenotype during regression of liver fibrosis. Proc Natl Acad Sci U S A 2012, 109:9448-9453

9. Troeger JS, Mederacke I, Gwak GY, Dapito DH, Mu X, Hsu CC, Pradere JP, Friedman RA, Schwabe RF: Deactivation of hepatic stellate cells during liver fibrosis resolution in mice. Gastroenterology 2012, 143:1073-1083.e22

10. Lukacs NW, Chensue SW, Smith RE, Strieter RM, Warmington K, Wilke C, Kunkel SL: Production of monocyte chemoattractant protein-1 and macrophage inflammatory protein-1 alpha by inflammatory granuloma fibroblasts. Am J Pathol 1994, 144:711-718 
11. Borthwick LA: The IL-1 cytokine family and its role in inflammation and fibrosis in the lung. Semin Immunopathol 2016, 38:517-534

12. Larsen CG, Anderson AO, Oppenheim JJ, Matsushima K: Production of interleukin-8 by human dermal fibroblasts and keratinocytes in response to interleukin-1 or tumour necrosis factor. Immunology 1989, 68:31-36

13. Flavell SJ, Hou TZ, Lax S, Filer AD, Salmon M, Buckley CD: Fibroblasts as novel therapeutic targets in chronic inflammation. Br J Pharmacol 2008, 153 Suppl 1:S241-S246

14. Borthwick LA, Suwara MI, Carnell SC, Green NJ, Mahida R, Dixon D, Gillespie CS, Cartwright TN, Horabin J, Walker A, Olin E, Rangar M, Gardner A, Mann J, Corris PA, Mann DA, Fisher AJ: Pseudomonas aeruginosa induced airway epithelial injury drives fibroblast activation: a mechanism in chronic lung allograft dysfunction. Am J Transplant 2016, 16:1751-1765

15. Suwara MI, Green NJ, Borthwick LA, Mann J, Mayer-Barber KD, Barron L, Corris PA, Farrow SN, Wynn TA, Fisher AJ, Mann DA: IL-1alpha released from damaged epithelial cells is sufficient and essential to trigger inflammatory responses in human lung fibroblasts. Mucosal Immunol 2014, 7:684-693

16. Wilson CL, Mann J, Walsh M, Perrugoria MJ, Oakley F, Wright MC, Brignole C, Di Paolo D, Perri P, Ponzoni M, Karin M, Mann DA: Quiescent hepatic stellate cells functionally contribute to the hepatic innate immune response via TLR3. PLoS One 2014, 9:e83391

17. Turner NA, Das A, Warburton P, O'Regan DJ, Ball SG, Porter KE: Interleukin-1alpha stimulates proinflammatory cytokine expression in human cardiac myofibroblasts. Am J Physiol Heart Circ Physiol 2009, 297:H1117-H1127

18. Meddeb M, Carpentier W, Cagnard N, Nadaud S, Grillon A, Barthel C, De Martino SJ, Jaulhac B, Boulanger N, Schramm F: Homogeneous inflammatory gene profiles induced in human dermal fibroblasts in response to the three main species of Borrelia burgdorferi sensu lato. PLoS One 2016, 11:e0164117

19. Dixon D, Coates J, del Carpio Pons A, Horabin J, Walker A, Abdul N, Kalson NS, Brewster NT, Weir DJ, Deehan DJ, Mann DA, Borthwick LA: A potential mode of action for Anakinra in patients with arthrofibrosis following total knee arthroplasty. Sci Rep 2015, 5:16466

20. Gollwitzer H, Burgkart R, Diehl P, Gradinger R, Buhren V: Therapy of arthrofibrosis after total knee arthroplasty. [German]. Orthopade 2006, 35:143-152

21. Petsche TS, Hutchinson MR: Loss of extension after reconstruction of the anterior cruciate ligament. J Am Acad Orthop Surg 1999, 7: $119-127$

22. Abdul N, Dixon D, Walker A, Horabin J, Smith N, Weir DJ, Brewster NT, Deehan DJ, Mann DA, Borthwick LA: Fibrosis is a common outcome following total knee arthroplasty. Sci Rep 2015, 5:16469

23. Ruppert M, Theiss C, Knoss P, Kendoff D, Krukemeyer MG, Schroder N, Brand-Saberi B, Gehrke T, Krenn V: Histopathological, immunohistochemical criteria and confocal laser-scanning data of arthrofibrosis. Pathol Res Pract 2013, 209:681-688

24. Bade MJ, Kohrt WM, Stevens-Lapsley JE: Outcomes before and after total knee arthroplasty compared to healthy adults. J Orthop Sports Phys Ther 2010, 40:559-567

25. Hofmann S, Seitlinger G, Djahani O, Pietsch M: The painful knee after TKA: a diagnostic algorithm for failure analysis. Knee Surg Sports Traumatol Arthrosc 2011, 19:1442-1452

26. Kalson NS, Borthwick LA, Mann DA, Deehan DJ, Lewis P, Mann C, Mont MA, Morgan-Jones R, Oussedik S, Williams FM, Toms A, Argenson JN, Bellemans J, Bhave A, Furnes O, Gollwitzer H, Haddad FS, Hofmann S, Krenn V: International consensus on the definition and classification of fibrosis of the knee joint. Bone Joint J 2016, 98-B:1479-1488

27. Parvizi J, Gehrke T, Chen AF: Proceedings of the International Consensus on Periprosthetic Joint Infection. Bone Joint J 2013, 95-B: $1450-1452$

28. Grau L, Gunder MA, Schneiderbauer M: Difficult-to-detect lowgrade infections responsible for poor outcomes in total knee arthroplasty. Am J Orthop (Belle Mead NJ) 2017, 46:E148-E153
29. Nodzo SR, Westrich GH, Henry MW, Miller AO: Clinical analysis of Propionibacterium acnes infection after total knee arthroplasty. J Arthroplasty 2016, 31:1986-1989

30. Xia J, Benner MJ, Hancock RE: NetworkAnalyst: integrative approaches for protein-protein interaction network analysis and visual exploration. Nucleic Acids Res 2014, 42:W167-W174

31. Breuer K, Foroushani AK, Laird MR, Chen C, Sribnaia A, Lo R, Winsor GL, Hancock RE, Brinkman FS, Lynn DJ: InnateDB: systems biology of innate immunity and beyond-recent updates and continuing curation. Nucleic Acids Res 2013, 41:D1228-D1233

32. Smoot ME, Ono K, Ruscheinski J, Wang PL, Ideker T: Cytoscape 2.8: new features for data integration and network visualization. Bioinformatics 2011, 27:431-432

33. Friedman J, Hastie T, Tibshirani R: Regularization paths for generalized linear models via coordinate descent. J Stat Softw 2010, 33: $1-22$

34. Robin X, Turck N, Hainard A, Tiberti N, Lisacek F, Sanchez JC, Muller M: pROC: an open-source package for $\mathrm{R}$ and $\mathrm{S}+$ to analyze and compare ROC curves. BMC Bioinformatics 2011, 12:77

35. Unterhauser FN, Bosch U, Zeichen J, Weiler A: Alpha-smooth muscle actin containing contractile fibroblastic cells in human knee arthrofibrosis tissue: winner of the AGA-DonJoy Award 2003. Arch Orthop Trauma Surg 2004, 124:585-591

36. Galli SJ, Tsai M, Piliponsky AM: The development of allergic inflammation. Nature 2008, 454:445-454

37. Hoesel B, Schmid JA: The complexity of NF-kappaB signaling in inflammation and cancer. Mol Cancer 2013, 12:86

38. Funakoshi-Tago M, Tago K, Sato Y, Tominaga S, Kasahara T: JAK2 is an important signal transducer in IL-33-induced NF-kappaB activation. Cell Signal 2011, 23:363-370

39. Freudlsperger C, Bian Y, Contag Wise S, Burnett J, Coupar J, Yang X, Chen Z, Van Waes C: TGF-beta and NF-kappaB signal pathway cross-talk is mediated through TAK1 and SMAD7 in a subset of head and neck cancers. Oncogene 2013, 32: $1549-1559$

40. Ye C, Zhang W, Wu W, Xu M, Nonso NS, He R: Influence of the infrapatellar fat pad resection during total knee arthroplasty: a systematic review and meta-analysis. PLoS One 2016, 11:e0163515

41. White L, Holyoak R, Sant J, Hartnell N, Mullan J: The effect of infrapatellar fat pad resection on outcomes post-total knee arthroplasty: a systematic review. Arch Orthop Trauma Surg 2016, 136:701-708

42. Scarpa M, Kessler S, Sadler T, West G, Homer C, McDonald C, de la Motte C, Fiocchi C, Stylianou E: The epithelial danger signal IL1alpha is a potent activator of fibroblasts and reactivator of intestinal inflammation. Am J Pathol 2015, 185:1624-1637

43. Buchan G, Barrett K, Turner M, Chantry D, Maini RN, Feldmann M: Interleukin-1 and tumour necrosis factor mRNA expression in rheumatoid arthritis: prolonged production of IL-1 alpha. Clin Exp Immunol 1988, 73:449-455

44. Firestein GS, Alvaro-Gracia JM, Maki R: Quantitative analysis of cytokine gene expression in rheumatoid arthritis. J Immunol 1990, 144:3347-3353

45. Deshmane SL, Kremlev S, Amini S, Sawaya BE: Monocyte chemoattractant protein-1 (MCP-1): an overview. J Interferon Cytokine Res 2009, 29:313-326

46. O'Connor T, Borsig L, Heikenwalder M: CCL2-CCR2 signaling in disease pathogenesis. Endocr Metab Immune Disord Drug Targets 2015, 15:105-118

47. Endres M, Andreas K, Kalwitz G, Freymann U, Neumann K, Ringe J, Sittinger M, Haupl T, Kaps C: Chemokine profile of synovial fluid from normal, osteoarthritis and rheumatoid arthritis patients: CCL25, CXCL10 and XCL1 recruit human subchondral mesenchymal progenitor cells. Osteoarthritis Cartil 2010, 18:1458-1466

48. Stankovic A, Slavic V, Stamenkovic B, Kamenov B, Bojanovic M, Mitrovic DR: Serum and synovial fluid concentrations of CCL2 (MCP1) chemokine in patients suffering rheumatoid arthritis and osteoarthritis reflect disease activity. Bratisl Lek Listy 2009, 110:641-646 
49. Watt FE, Paterson E, Freidin A, Kenny M, Judge A, Saklatvala J, Williams A, Vincent TL: Acute molecular changes in synovial fluid following human knee injury: association with early clinical outcomes. Arthritis Rheumatol 2016, 68:2129-2140

50. Struglics A, Larsson S, Kumahashi N, Frobell R, Lohmander LS: Changes in cytokines and aggrecan ARGS neoepitope in synovial fluid and serum and in C-terminal crosslinking telopeptide of type II collagen and $\mathrm{N}$-terminal crosslinking telopeptide of type I collagen in urine over five years after anterior cruciate ligament rupture: an exploratory analysis in the knee anterior cruciate ligament, nonsurgical versus surgical treatment trial. Arthritis Rheumatol 2015, 67: $1816-1825$

51. Raghu H, Lepus CM, Wang Q, Wong HH, Lingampalli N, Oliviero F, Punzi L, Giori NJ, Goodman SB, Chu CR, Sokolove JB, Robinson WH: CCL2/CCR2, but not CCL5/CCR5, mediates monocyte recruitment, inflammation and cartilage destruction in osteoarthritis. Ann Rheum Dis 2017, 76: 914-922

52. Sohn DH, Sokolove J, Sharpe O, Erhart JC, Chandra PE, Lahey LJ, Lindstrom TM, Hwang I, Boyer KA, Andriacchi TP, Robinson WH: Plasma proteins present in osteoarthritic synovial fluid can stimulate cytokine production via Toll-like receptor 4. Arthritis Res Ther 2012, 14:R7

53. Menetski J, Mistry S, Lu M, Mudgett JS, Ransohoff RM, Demartino JA, Macintyre DE, Abbadie C: Mice overexpressing chemokine ligand 2 (CCL2) in astrocytes display enhanced nociceptive responses. Neuroscience 2007, 149:706-714

54. White FA, Sun J, Waters SM, Ma C, Ren D, Ripsch M, Steflik J, Cortright DN, Lamotte RH, Miller RJ: Excitatory monocyte chemoattractant protein-1 signaling is up-regulated in sensory neurons after chronic compression of the dorsal root ganglion. Proc Natl Acad Sci U S A 2005, 102:14092-14097

55. White FA, Bhangoo SK, Miller RJ: Chemokines: integrators of pain and inflammation. Nat Rev Drug Discov 2005, 4:834-844

56. Ji RR, Xu ZZ, Gao YJ: Emerging targets in neuroinflammationdriven chronic pain. Nat Rev Drug Discov 2014, 13:533-548

57. Miotla Zarebska J, Chanalaris A, Driscoll C, Burleigh A, Miller RE, Malfait AM, Stott B, Vincent TL: CCL2 and CCR2 regulate painrelated behaviour and early gene expression in post-traumatic murine osteoarthritis but contribute little to chondropathy. Osteoarthritis Cartilage 2017, 25:406-412

58. Brown CA, Toth AP, Magnussen B: Clinical benefits of intraarticular anakinra for arthrofibrosis. Orthopedics 2010, 33:877

59. Gupta SC, Sundaram C, Reuter S, Aggarwal BB: Inhibiting NFkappaB activation by small molecules as a therapeutic strategy. Biochim Biophys Acta 2010, 1799:775-787

60. Gilmore TD, Herscovitch M: Inhibitors of NF-kappaB signaling: 785 and counting. Oncogene 2006, 25:6887-6899

61. Beg AA, Baltimore D: An essential role for NF-kappaB in preventing TNF-alpha-induced cell death. Science 1996, 274:782-784 